\title{
Supersymmetry algebra in super Yang-Mills theories
}

\author{
Shuichi Yokoyama \\ Physics Department, Technion - Israel Institute of Technology, \\ Technion City - Haifa 3200003, Israel \\ E-mail: shuichitoissho@gmail.com
}

ABSTRACT: We compute supersymmetry algebra (superalgebra) in supersymmetric YangMills theories (SYM) consisting of a vector multiplet including fermionic contribution in six dimensions. We show that the contribution of fermion is given by boundary terms. From six dimensional results we determine superalgebras of five and four dimensional SYM by dimensional reduction. In five dimensional superalgebra the Kaluza-Klein momentum and the instanton particle charge are not the same but algebraically indistinguishable. We also extend this calculation including a hyper multiplet and for maximally SYM. We derive extended supersymmetry algebras in these four dimensional SYM with the holomorphic coupling constant given in hep-th/9408099.

KeYwords: Supersymmetric gauge theory, Extended Supersymmetry, Field Theories in Higher Dimensions

ARXIV EPRINT: 1506.03522 


\section{Contents}

1 Introduction $\quad 1$

2 Superalgebra in 10d SYM 2

3 Superalgebra in 6d SYM $\quad 6$

3.1 Vector multiplet $\quad 6$

3.2 Inclusion of a hyper multiplet 9

$3.36 \mathrm{~d} \mathcal{N}=2$ superalgebra 11

4 Superalgebra in 5d SYM $\quad 14$

$\begin{array}{lll}4.1 & \text { Vector multiplet } & 14\end{array}$

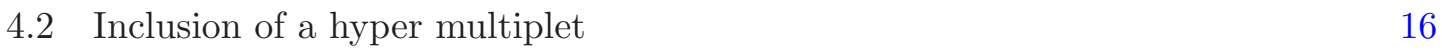

$\begin{array}{ll}4.35 \mathrm{~d} \mathcal{N}=2 \text { superalgebra } & 17\end{array}$

5 Superalgebra in 4d SYM 20

$\begin{array}{ll}5.1 \mathcal{N}=2 \text { vector multiplet } & 20\end{array}$

5.2 Inclusion of a hyper multiplet 23

$\begin{array}{ll}5.3 \mathcal{N}=4 \text { superalgebra } & 26\end{array}$

6 Discussion 28

$\begin{array}{ll}\text { A A formula of gamma matrix } & 29\end{array}$

B Convention in six dimensions $\quad 30$

\section{Introduction}

Supersymmetric Yang-Mills theories (SYM) in higher dimensions than four [1] have been uncovered to possess their own rich structure of supersymmetric (SUSY) quantum field theories (QFT) in spite of their nature of lack of power counting renormalizability.

In five dimensional case the structure of Coulomb branch at long distance can be determined exactly due to the fact that prepotential can be computed exactly by oneloop $[2,3]$. What was interestingly found is that if the number of matter multiplets is small enough, there is no singularity of Landau pole and it becomes possible to take strong coupling limit on smooth moduli space, which leads to an ultra-violet (UV) fixed point with global symmetry enhancement depending on the matter content. This phenomenon has been further studied by using brane construction [4-8], a superconformal index [9-13] and direct state analysis [14-16]. 
Maximally SYM in five dimensions has also attracted a great deal of attention and studied in relation to six dimensional $(2,0)$ superconformal field theory (SCFT) [17, 18], whose Lagrangian description is unknown. Although it was shown that UV divergence of five dimensional SYM appears at six loops [19], which indicates necessity of UV completion, BPS sector of the theory is expected to encode information of that of $(2,0)$ SCFT due to its insensitivity to UV. It was shown that five dimensional maximally SYM contains Kaluza-Klein modes coming from the sixth direction as states with instanton-particle charge [18, 20, 21].

Search of a SUSY gauge theory enjoying a non-trivial UV fixed point has also been done in six dimensions [22]. The requirement is gauge anomaly cancellation as is the case in even dimensional QFT. It has been shown that anomaly of matter multiplets can cancel if the number is small enough for $\mathrm{SU}(2)$ gauge group. This was further studied in other simple gauge groups [23]. Examples of nontrivial UV fixed points are provided by compactification of string theory with strong coupling (or tensionless) limit [24-27]. See [28] for other examples of six dimensional gauge theories.

In comparison to these non-trivial developments of higher dimensional SUSY gauge theories this paper performs a basic calculation for an aim to determine supersymmetry algebra (superalgebra) of six dimensional SYM. Lagrangian description allows us to compute six dimensional superalgebra explicitly and dimensional reduction for the six dimensional result enables us to compare the Kaluza-Klein momentum of the sixth direction and instantonparticle charge, which are identified in earlier study. We also recover a basic result of superalgebra of four dimensional $\mathcal{N}=2 \mathrm{SYM}$ including a hyper multiplet, which leads to the formula of central charge with the holomorphic coupling constant insightfully chosen in [29].

The rest of this paper is organized as follows. In section 2 we review the method to determine superalgebra by using ten dimensional SYM following [30]. In section 3 we compute superalgebra of SYM in six dimensions including contribution of a hyper mutliplet (section 3.2). In particular the $\mathcal{N}=2$ algebra in six dimensions is determined by dimensional reduction of ten dimensional one. In section 4 , section 5 we determine superalgebras of five and four dimensional SYM, respectively, by dimensional reduction from six or ten dimensions. Section 6 is devoted to conclusion and discussion. Appendix contains a formula of gamma matrix (appendix A) and convention in six dimensions used in this paper (appendix B).

\section{Superalgebra in 10d SYM}

In this section we review the supersymmetry algebra in ten dimensional supersymmetric Yang-Mills theory [30] using our convention. Results in this section are used to derive similar results of maximally SYM in other dimensions by dimensional reduction later. The fields of SYM in ten dimensions are a gauge field $A_{\mathbf{M}}(\mathbf{M}=0,1, \cdots, 9)^{1}$ and a MajoranaWeyl fermion (gaugino) $\lambda$, whose chirality we choose as positive.

$$
\hat{\Gamma}_{10} \lambda=\lambda, \quad \lambda=C_{10} \bar{\lambda}^{T},
$$

\footnotetext{
${ }^{1}$ The gauge field in this paper is anti-hermitian.
} 
where $\Gamma_{\mathbf{M}}$ are $\mathrm{SO}(1,9)$ gamma matrices, $\bar{\lambda}=i \lambda^{\dagger} \Gamma_{0}$,

$$
\hat{\Gamma}_{10}=\Gamma_{01 \ldots 9}, \quad C_{10}=-\Gamma_{03579} .
$$

We realize the ten dimensional gamma matrices by using six dimensional ones as (3.45) in section 3.3, which is useful for dimensional reduction carried out later. We employ matrix notation for spinor indices and ${ }^{T}$ acts only on them. The SYM Lagrangian (density) in ten dimensions is given by

$$
\mathcal{L}_{10}=\frac{1}{g_{10}^{2}} \operatorname{Tr}\left[\frac{1}{4} F_{\mathbf{M N}} F^{\mathbf{M N}}+\frac{1}{2} \bar{\lambda} \Gamma^{\mathbf{M}} D_{\mathbf{M}} \lambda\right]
$$

where $F_{\mathbf{M N}}=\left[D_{\mathbf{M}}, D_{\mathbf{N}}\right], D_{\mathbf{M}}=\partial_{\mathbf{M}}+A_{\mathbf{M}}$. The action constructed from this Lagrangian is invariant under supersymmetry transformation rule given by

$$
\Delta A_{\mathbf{M}}=\bar{\epsilon} \Gamma_{\mathbf{M}} \lambda, \quad \Delta \lambda=\frac{1}{2} F_{\mathbf{M N}} \Gamma^{\mathbf{M N}} \epsilon
$$

where $\epsilon$ is a supersymmetry parameter of Majorana-Weyl fermion satisfying $\hat{\Gamma}_{10} \epsilon=\epsilon$ and $C_{10} \bar{\epsilon}^{T}=-\epsilon$. The supersymmetry current is obtained as

$$
\overline{S^{\mathbf{P}}}=\frac{1}{g_{10}^{2}} \operatorname{Tr}\left[\bar{\lambda} \frac{1}{2} F_{\mathbf{M N}} \Gamma^{\mathbf{P}} \Gamma^{\mathbf{M N}}\right], \quad S^{\mathbf{P}}=\frac{1}{g_{10}^{2}} \operatorname{Tr}\left[-\frac{1}{2} F_{\mathbf{M N}} \Gamma^{\mathbf{M N}} \Gamma^{\mathbf{P}} \lambda\right]
$$

where the SUSY current satisfies $\overline{S^{\mathbf{P}}} \epsilon=\bar{\epsilon} S^{\mathbf{P}}$.

To compute the supersymmetry algebra of this theory, we compute variation of the SUSY current under supersymmetry transformation.

$$
2 g_{10}^{2} \Delta \overline{S^{\mathbf{P}}}=\operatorname{Tr}\left[\Delta \bar{\lambda} F_{\mathbf{M N}} \Gamma^{\mathbf{P}} \Gamma^{\mathbf{M N}}+2 \bar{\lambda} D_{\mathbf{M}} \Delta A_{\mathbf{N}} \Gamma^{\mathbf{P}} \Gamma^{\mathbf{M N}}\right] .
$$

The 1st term can be calculated as

$$
\operatorname{Tr}\left[\Delta \bar{\lambda} F_{\mathbf{M N}} \Gamma^{\mathbf{P}} \Gamma^{\mathbf{M N}}\right]=-\frac{1}{2} \operatorname{Tr}\left[F_{\mathbf{Q R}} F_{\mathbf{M N}}\right] \bar{\epsilon} \Gamma^{\mathbf{R Q P M N}}-4 \operatorname{Tr}\left[F^{\mathbf{P M}} F_{\mathbf{M N}}\right] \bar{\epsilon} \Gamma^{\mathbf{N}}-\operatorname{Tr}\left[F^{\mathbf{M N}} F_{\mathbf{M N}}\right] \bar{\epsilon} \Gamma^{\mathbf{P}}
$$

The 2 nd term is calculated as follows.

$$
\begin{aligned}
& \operatorname{Tr}\left[2 \bar{\lambda} D_{\mathbf{M}} \Delta A_{\mathbf{N}} \Gamma^{\mathbf{P}} \Gamma^{\mathbf{M N}}\right]=2 \operatorname{Tr}\left[\bar{\epsilon} \Gamma_{\mathbf{N}} D_{\mathbf{M}} \lambda \bar{\lambda} \Gamma^{\mathbf{P}} \Gamma^{\mathbf{M N}}\right] \\
& =\frac{-1}{8}\left(\operatorname{Tr}\left[\bar{\lambda} \Gamma^{\mathbf{Q}} D_{\mathbf{M}} \lambda\right] \bar{\epsilon} \Gamma_{\mathbf{N}} \Gamma_{\mathbf{Q}} \Gamma^{\mathbf{P}} \Gamma^{\mathbf{M N}}+\frac{1}{3 !} \operatorname{Tr}\left[\bar{\lambda} \Gamma^{\mathbf{Q R S}} D_{\mathbf{M}} \lambda\right] \bar{\epsilon} \Gamma_{\mathbf{N}} \Gamma_{\mathbf{S R Q}} \Gamma^{\mathbf{P}} \Gamma^{\mathbf{M N}}\right. \\
& \left.\quad+\frac{1}{2 \cdot 5 !} \operatorname{Tr}\left[\bar{\lambda} \Gamma^{\mathbf{Q R S T U}} D_{\mathbf{M}} \lambda\right] \bar{\epsilon} \Gamma_{\mathbf{N}} \Gamma_{\mathbf{U T S R Q}} \Gamma^{\mathbf{P}} \Gamma^{\mathbf{M N}}\right),
\end{aligned}
$$

in which we used ten dimensional Fierz identity

$$
\chi \bar{\psi}=\frac{-1}{2^{4}}\left(\bar{\psi} \Gamma^{\mathbf{M}} \chi \Gamma_{\mathbf{M}}+\frac{1}{3 !} \bar{\psi} \Gamma^{\mathbf{M N P}} \chi \Gamma_{\mathbf{P N M}}+\frac{1}{2 \cdot 5 !} \bar{\psi} \Gamma^{\mathbf{M N P Q R}} \chi \Gamma_{\mathbf{R Q P N M}}\right) \frac{1-(-)^{\psi} \hat{\Gamma}_{10}}{2}
$$


where $\psi, \chi$ are Weyl fermions of the same chirality and we denote the chirality of $\psi$ by $(-)^{\psi}$. By using the equation of motion of gaugino $\Gamma^{\mathbf{M}} D_{\mathbf{M}} \lambda=0$ and a formula

$$
\Gamma^{\mathbf{M}_{1} \cdots \mathbf{M}_{5}} \psi \bar{\chi} \Gamma_{\mathbf{M}_{1} \cdots \mathbf{M}_{5}}=0
$$

where $\psi$ and $\chi$ are Weyl fermion with the same chirality, the above can be simplified as

$$
(2.8)=\operatorname{Tr}\left[\bar{\lambda} \Gamma_{\mathbf{M}_{1}} D_{\mathbf{M}_{2}} \lambda\right] \bar{\epsilon} \Gamma^{\mathbf{M}_{2} \mathbf{M}_{1} \mathbf{P}}+2 \operatorname{Tr}\left[\bar{\lambda} \Gamma^{\mathbf{P}} D_{\mathbf{M}} \lambda\right] \bar{\epsilon} \Gamma^{\mathbf{M}}+\operatorname{Tr}\left[\bar{\lambda} \Gamma^{\mathbf{P}}{ }_{\mathbf{M}_{1} \mathbf{M}_{2}} D_{\mathbf{M}_{3}} \lambda\right] \bar{\epsilon} \Gamma^{\mathbf{M}_{3} \mathbf{M}_{2} \mathbf{M}_{1}} .
$$

Summing up these terms we find ${ }^{2}$

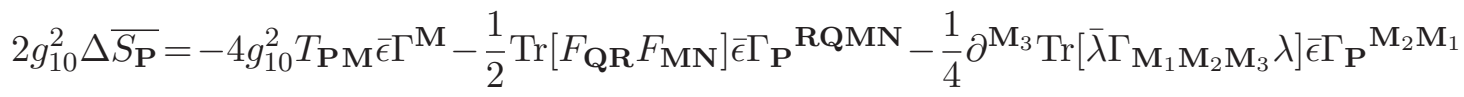

$$
\begin{aligned}
& +\frac{1}{2} \partial_{\mathbf{M}_{3}} \operatorname{Tr}\left[\bar{\lambda} \Gamma_{\mathbf{P M}_{1} \mathbf{M}_{2}} \lambda\right] \bar{\epsilon} \Gamma^{\mathbf{M}_{3} \mathbf{M}_{2} \mathbf{M}_{1}}-\frac{1}{2} \partial^{\mathbf{N}} \operatorname{Tr}\left[\bar{\lambda} \Gamma_{\mathbf{P M N}} \lambda\right] \bar{\epsilon} \Gamma^{\mathbf{M}}
\end{aligned}
$$

where $T_{\mathbf{P M}}$ is the stress tensor given by

$$
T_{\mathbf{M P}}=\frac{1}{4 g_{10}^{2}}\left(4 \operatorname{Tr}\left[F_{\mathbf{P}^{\mathbf{N}}} F_{\mathbf{N M}}\right]+\eta_{\mathbf{M P}} \operatorname{Tr}\left[F_{\mathbf{M N}} F^{\mathbf{M N}}\right]-2 \operatorname{Tr}\left[\bar{\lambda} \Gamma_{(\mathbf{M}} D_{\mathbf{P})} \lambda\right]\right)
$$

and we used $\bar{\lambda} \Gamma_{\mathbf{M}_{1} \mathbf{M}_{2} \mathbf{M}_{3}} D_{\mathbf{M}} \lambda=\frac{1}{2} D_{\mathbf{M}}\left(\bar{\lambda} \Gamma_{\mathbf{M}_{1} \mathbf{M}_{2} \mathbf{M}_{3}} \lambda\right)$, and $X_{(A} Y_{B)}:=\frac{1}{2}\left(X_{A} Y_{B}+X_{B} Y_{A}\right)$.

The supercharge is defined by

$$
Q=\int d^{9} x S^{0}
$$

Under the standard convention of canonical formalism, it can be shown that

$$
\Delta \mathcal{O}=[-i \bar{\epsilon} Q, \mathcal{O}]
$$

for a gauge invariant operator $\mathcal{O}$ and a canonical bracket. Although it is not difficult to show this computationally, it needs a little careful argument to justify this, as we shall do below. The canonical momentum of the gaugino is computed as

$$
\Pi_{\lambda}=\frac{\partial \mathcal{L}}{\partial\left(\partial_{0} \lambda\right)}=\frac{1}{2 g_{10}^{2}}\left[-\bar{\lambda} \Gamma^{0}\right]
$$

Under the canonical commutation relation $\left[\Pi_{\lambda}, \lambda\right]=i \delta$, where $\delta$ is the unit matrix in terms of implicit space, gauge and spinor indices, one can easily show that

$$
\Delta \lambda=[-i \bar{\epsilon} Q, \lambda]
$$

On the other hand, the canonical momentum of the gauge field is computed as

$$
\Pi_{A_{\mathbf{M}}}=\frac{\partial \mathcal{L}}{\partial\left(\partial_{0} A_{\mathbf{M}}\right)}=\frac{1}{g_{10}^{2}} F^{0 \mathbf{M}},
$$

\footnotetext{
${ }^{2}$ Our result in the fermionic part is different from that in [30]. One of the reasons is that the stress tensor given in [30] is not a symmetric one in the fermionic part. However the argument there does not need modification since the fermionic part was neglected in other parts of that paper.
} 
which has a vanishing component for time direction as ordinary Yang-Mills theory. This suggests that there is no kinetic term of the time component of the gauge field in the (offshell) Lagrangian and the system is constrained by saddle point equation thereof, which is given by $D_{M} \Pi^{M}=0$, where $M$ runs the space directions. This requires us to choose a set of dynamical (or canonical) variables to quantize the system. We naturally choose it as the gauge fields of the space directions. Then the canonical commutation relation is $\left[\Pi_{A_{M}}, A_{N}\right]=i \delta_{N}^{M} \delta$. By using this it is not difficult to show that

$$
\Delta A_{M}=\left[-i \bar{\epsilon} Q, A_{M}\right]
$$

We stress that the SUSY variation (2.4) is reproduced for the dynamical gauge fields $\left(A_{M}\right)$ and not for the auxiliary one $\left(A_{0}\right) .{ }^{3}$ This argument is consistent with the fact that the SUSY variation of supercurrent derived in (2.12) is an on-shell relation. One may ask that there will be another constraint by fixing gauge symmetry which every Yang-Mills theory possesses, in which case one has to use not the canonical bracket but a Dirac one for (2.19) in order to be consistent with the gauge fixing. This should be the case though we still claim that (2.15) holds for a canonical bracket. The argument is as follows. When one fixes gauge symmetry, the initial supersymmetry transformation is not consistent with the fixed gauge in general. One can modify the SUSY transformation so as to be consistent with the gauge fixing by combining gauge transformation. Then the right-hand side of (2.19) replaced by the Dirac bracket will reproduce the modified SUSY transformation for the gauge fields. This suggests that the modified SUSY transformation for a gauge invariant operator should agree with the initial one because the modification is given by a gauge transformation. Thus one has only to use a canonical bracket and do not need to use a Dirac one in (2.15).

As a result, by using (2.12) and (2.15), algebra between the supercurrent and supercharge in SYM in ten dimensions (local form of SUSY algebra) is given by

$$
\begin{aligned}
& \left\{Q, \overline{S_{\mathbf{P}}}\right\}=-2 i T_{\mathbf{P M}} \Gamma^{\mathbf{M}}+J_{\mathbf{P M}} \Gamma^{\mathbf{M}}+J_{\mathbf{P M}_{1} \mathbf{M}_{2} \mathbf{M}_{3}} \Gamma^{\mathbf{M}_{3} \mathbf{M}_{2} \mathbf{M}_{1}}+i C_{\mathbf{M}_{1} \mathbf{M}_{2}} \Gamma_{\mathbf{P}} \mathbf{M}_{2} \mathbf{M}_{1} \\
& +J_{\mathbf{P}} \mathbf{M}_{5} \mathbf{M}_{4} \mathbf{M}_{3} \mathbf{M}_{2} \mathbf{M}_{1} \Gamma_{\mathbf{M}_{1} \mathbf{M}_{2} \mathbf{M}_{3} \mathbf{M}_{4} \mathbf{M}_{5}}
\end{aligned}
$$

where we define

$$
\begin{aligned}
J_{\mathbf{P M}} & =\frac{-i}{4 g_{10}^{2}} \partial^{\mathbf{N}} \operatorname{Tr}\left[\bar{\lambda} \Gamma_{\mathbf{P M N}} \lambda\right] \\
C_{\mathbf{M}_{1} \mathbf{M}_{2}} & =-\frac{1}{8 g_{10}^{2}} \partial^{\mathbf{M}_{3}} \operatorname{Tr}\left[\bar{\lambda} \Gamma_{\left.\mathbf{M}_{1} \mathbf{M}_{2} \mathbf{M}_{3} \lambda\right]}\right. \\
J_{\mathbf{P M}_{1} \mathbf{M}_{2} \mathbf{M}_{3}} & =\frac{i}{4 g_{10}^{2}} \partial_{\mathbf{M}_{3}} \operatorname{Tr}\left[\bar{\lambda} \Gamma_{\left.\mathbf{P M}_{1} \mathbf{M}_{2} \lambda\right]}\right. \\
J_{\mathbf{P}} \mathbf{M}_{5} \mathbf{M}_{4} \mathbf{M}_{3} \mathbf{M}_{2} \mathbf{M}_{1} & =-\frac{i}{4 g_{10}^{2}} \operatorname{Tr}\left[F_{\mathbf{Q R}} F_{\mathbf{M N}}\right] \varepsilon_{\mathbf{P}} \mathbf{Q R M N M}_{5} \mathbf{M}_{4} \mathbf{M}_{3} \mathbf{M}_{2} \mathbf{M}_{1}
\end{aligned}
$$

\footnotetext{
${ }^{3}$ This standpoint may be different from one argued in [31], where SUSY algebra of a general four dimensional $\mathcal{N}=2 \mathrm{SYM}$ of an $\mathcal{N}=2$ vector multiplet was studied. It seems there that the SUSY variation of all the components of the gauge fields was reproduced in appendix D, which may be incorrect for that of the auxiliary gauge field.
} 
with $\varepsilon_{01 \ldots 9}=1$. Note that the contributions of fermions are total derivative terms. Especially we obtain supersymmetry algebra in ten dimensional SYM as

$$
\{Q, \bar{Q}\}=-2 i P_{\mathbf{M}} \Gamma^{\mathbf{M}}+Z_{\mathbf{M}} \Gamma^{\mathbf{M}}+Z_{\mathbf{M}_{1} \mathbf{M}_{2} \mathbf{M}_{3}} \Gamma^{\mathbf{M}_{3} \mathbf{M}_{2} \mathbf{M}_{1}}+Z_{\mathbf{M}_{5} \mathbf{M}_{4} \mathbf{M}_{3} \mathbf{M}_{2} \mathbf{M}_{1}} \Gamma^{\mathbf{M}_{1} \mathbf{M}_{2} \mathbf{M}_{3} \mathbf{M}_{4} \mathbf{M}_{5}}
$$

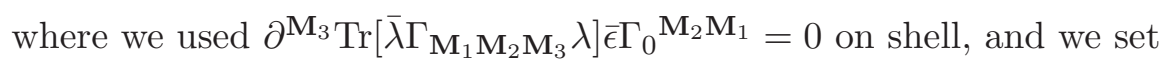

$$
\begin{aligned}
P^{\mathbf{M}} & =\int d^{9} x T^{0 \mathbf{M}}, & Z^{\mathbf{M}} & =\int d^{9} x J^{0 \mathbf{M}} \\
Z^{\mathbf{M}_{1} \mathbf{M}_{2} \mathbf{M}_{3}} & =\int d^{9} x J^{0 \mathbf{M}_{1} \mathbf{M}_{2} \mathbf{M}_{3}}, & Z^{\mathbf{M}_{5} \mathbf{M}_{4} \mathbf{M}_{3} \mathbf{M}_{2} \mathbf{M}_{1}} & =\int d^{9} x J^{0 \mathbf{M}_{5} \mathbf{M}_{4} \mathbf{M}_{3} \mathbf{M}_{2} \mathbf{M}_{1}} .
\end{aligned}
$$

\section{Superalgebra in 6d SYM}

In this section we compute supersymmetry algebra of six dimensional SYM with eight and sixteen supercharges. We derive results of maximally SYM in six dimensions by dimensional reduction of ten dimensional one obtained in the previous section.

\section{$3.1 \quad$ Vector multiplet}

First we consider a vector multiplet. This theory has $\mathrm{SU}(2) \simeq \mathrm{Sp}(1)$ global symmetry. The bosonic field contents are a gauge field $A_{M}, M=0,1, \cdots, 5$ and the $\mathrm{SU}(2)$ triplet auxiliary fields $D_{B}^{A}$ which satisfy $\left(D_{B}^{A}\right)^{\dagger}=D_{A}^{B}, D_{A}^{A}=0$. The super partner $\lambda^{A}$ is a $\operatorname{Sp}(1)$-Majorana Weyl fermion satisfying

$$
\hat{\Gamma} \lambda^{A}=+\lambda^{A}, \quad \varepsilon^{A B} C_{6}\left(\overline{\lambda^{B}}\right)^{T}=\lambda^{A}
$$

where $\varepsilon_{12}=\varepsilon^{12}=1$, and $\hat{\Gamma}$ and $C_{6}$ are a chirality matrix and a charge conjugation in six dimensions, respectively, defined by

$$
\hat{\Gamma}=\Gamma_{012345}, \quad C_{6}=\Gamma_{035}
$$

See appendix B for more details on our convention in six dimensions. In this convention, the supersymmetric Lagrangian reads

$$
\mathcal{L}_{V}=\frac{1}{g_{6}^{2}} \operatorname{Tr}\left[\frac{1}{4} F_{M N} F^{M N}+\frac{1}{2} \overline{\lambda^{A}} \Gamma^{M}\left[D_{M}, \lambda^{A}\right]+\frac{1}{2} D^{A}{ }_{B} D^{B}{ }_{A}\right]
$$

where $F_{M N}=\left[D_{M}, D_{N}\right], D_{M}=\partial_{M}+A_{M}$. The supersymmeric transformation rule is

$$
\begin{aligned}
\Delta A_{M} & =\overline{\epsilon^{A}} \Gamma_{M} \lambda^{A} \\
\Delta \lambda^{A} & =\frac{1}{2} F_{M N} \Gamma^{M N} \epsilon^{A}+\alpha D_{B}^{A} \epsilon^{B}, \\
\Delta D_{B}^{A} & =\alpha\left(D_{M} \overline{\lambda^{B}} \Gamma^{M} \epsilon^{A}-\frac{1}{2} \delta_{B}^{A} D_{M} \overline{\lambda^{C}} \Gamma^{M} \epsilon^{C}\right),
\end{aligned}
$$

where $\epsilon^{A}$ is also a symplectic-Majorana Weyl fermion such that $\hat{\Gamma} \epsilon^{A}=\epsilon^{A}$ and $\varepsilon^{A B} C_{6}\left(\overline{\epsilon^{B}}\right)^{T}=-\epsilon^{A}$. Thus the type of SUSY is $(1,0) . \alpha$ is arbitrary parameter and thus 
one can set to zero as long as one considers only vector multiplet due to the fact that the auxiliary field can be integrated out to be zero. Once one introduces coupling to a hyper multiplet, which is done in the next subsection, $\alpha$ is uniquely determined as $\alpha=\sqrt{2}$. The supersymmetry current of this theory is computed in the same way as in ten dimensions.

$$
\overline{S_{P}^{A}}=\frac{1}{g_{6}^{2}} \operatorname{Tr}\left[\frac{1}{2} \overline{\lambda^{A}} F_{M N} \Gamma_{P} \Gamma^{M N}\right], \quad S_{P}^{A}=\frac{1}{g_{6}^{2}} \operatorname{Tr}\left[-\frac{1}{2} F_{M N} \Gamma^{M N} \Gamma_{P} \lambda^{A}\right],
$$

where they are determined so as to satisfy $\overline{\epsilon^{A}} S_{P}^{A}=\overline{S_{P}^{A}} \epsilon^{A}$.

Let us compute the SUSY algebra of $\mathcal{N}=1 \mathrm{SYM}$ consisting of a vector multiplet.

$$
2 g_{6}^{2} \Delta_{\epsilon} \overline{S_{P}^{A}}=\operatorname{Tr}\left[\Delta \overline{\lambda^{A}} F_{M N} \Gamma_{P} \Gamma^{M N}+2 \overline{\lambda^{A}} D_{M} \Delta A_{N} \Gamma_{P} \Gamma^{M N}\right] .
$$

The 1st term can be calculated as

$$
\begin{aligned}
\operatorname{Tr}\left[\Delta \overline{\lambda^{A}} F_{M N} \Gamma_{P} \Gamma^{M N}\right]= & -\frac{1}{2} \operatorname{Tr}\left[F_{Q R} F_{M N}\right] \overline{\epsilon^{A}} \Gamma^{R Q}{ }_{P}^{M N}-4 \operatorname{Tr}\left[F_{P}{ }^{M} F_{M N}\right] \overline{\epsilon^{A}} \Gamma^{N}-\operatorname{Tr}\left[F^{M N} F_{M N}\right] \overline{\epsilon^{A}} \Gamma_{P} \\
& -\alpha \operatorname{Tr}\left[\left(D_{B}^{A}\right)^{\dagger} F_{M N}\right] \overline{\epsilon^{B}}\left(\Gamma_{P}{ }^{M N}+\delta_{P}^{M} \Gamma^{N}-\delta_{P}^{N} \Gamma^{M}\right) .
\end{aligned}
$$

The 2nd term is calculated as follows.

$$
\begin{aligned}
\operatorname{Tr}\left[2 \overline{\lambda^{A}} D_{M} \Delta A_{N} \Gamma_{P} \Gamma^{M N}\right]= & 2 \operatorname{Tr}\left[\overline{\epsilon^{B}} \Gamma_{N} D_{M} \lambda^{B} \overline{\lambda^{A}} \Gamma_{P} \Gamma^{M N}\right] \\
= & \frac{-1}{2} \operatorname{Tr}\left[\overline{\lambda^{A}} \Gamma^{M_{1}} D_{M} \lambda^{B}\right] \overline{\epsilon^{B}} \Gamma_{N} \Gamma_{M_{1}} \Gamma_{P} \Gamma^{M N} \\
& -\frac{1}{24} \operatorname{Tr}\left[\overline{\lambda^{A}} \Gamma^{M_{1} M_{2} M_{3}} D_{M} \lambda^{B}\right] \overline{\epsilon^{B}} \Gamma_{N} \Gamma_{M_{3} M_{2} M_{1} \Gamma_{P} \Gamma^{M N}}
\end{aligned}
$$

where we used a Fierz identity

$$
\chi \bar{\psi}=\frac{-1}{2^{2}}\left(\bar{\psi} \Gamma^{M} \chi \Gamma_{M}+\frac{1}{3 ! \cdot 2} \bar{\psi} \Gamma^{M N P} \chi \Gamma_{P N M}\right) \frac{1-(-)^{\psi} \hat{\Gamma}}{2}
$$

for Weyl fermions $\psi, \chi$ with the same chirality. By using

$$
\Gamma^{M_{1} M_{2} M_{3}} \psi \bar{\chi} \Gamma_{M_{1} M_{2} M_{3}}=0
$$

where $\psi$ and $\chi$ are Weyl fermions with the same chirality, and

$$
\overline{\lambda^{A}} \Gamma_{P} D_{M} \lambda^{B}=\frac{1}{2} D_{M}\left(\overline{\lambda^{A}} \Gamma_{P} \lambda_{B}\right)+\frac{1}{2} \delta_{A}^{B} \overline{\lambda^{C}} \Gamma_{P} D_{M} \lambda^{C}
$$

we find

$$
\operatorname{Tr}\left[2 \overline{\lambda^{A}} D_{M} \Delta A_{N} \Gamma_{P} \Gamma^{M N}\right]=2 \partial_{M} \operatorname{Tr}\left[\overline{\lambda^{A}} \Gamma_{P} \lambda^{B}\right] \overline{\epsilon^{B}} \Gamma^{M}+2 \operatorname{Tr}\left[\overline{\lambda^{C}} \Gamma_{P} D_{M} \lambda^{C}\right] \overline{\epsilon^{A}} \Gamma^{M}
$$

where we also used the equation of motion of gaugino. Collecting these we obtain

$$
\begin{aligned}
2 g_{6}^{2} \Delta \overline{S_{P}^{A}}= & -\frac{1}{2} \operatorname{Tr}\left[F_{Q R} F_{M N}\right] \overline{\epsilon^{A}} \Gamma^{R Q} P_{P}^{M N}-4 \operatorname{Tr}\left[F_{P}^{M} F_{M N}\right] \overline{\epsilon^{A}} \Gamma^{N}-\operatorname{Tr}\left[F^{M N} F_{M N}\right] \overline{\epsilon^{A}} \Gamma_{P} \\
& -\alpha \operatorname{Tr}\left[\left(D^{A}{ }^{\dagger} F_{M N}\right] \overline{\epsilon^{B}}\left(\Gamma_{P}{ }^{M N}+2 \delta_{P}^{M} \Gamma^{N}\right)\right. \\
& +2 \partial_{M} \operatorname{Tr}\left[\overline{\lambda^{A}} \Gamma_{P} \lambda^{B}\right] \overline{\epsilon^{B}} \Gamma^{M}+2 \operatorname{Tr}\left[\overline{\lambda^{C}} \Gamma_{P} D_{M} \lambda^{C}\right] \overline{\epsilon^{A}} \Gamma^{M} .
\end{aligned}
$$


Integrating out the auxiliary field gives $D_{B}^{A}=0$. Then

$$
\begin{aligned}
2 g_{6}^{2} \Delta \overline{S_{P}^{A}}= & -4 g_{6}^{2} T_{P M} \overline{\epsilon^{A}} \Gamma^{M}-\frac{1}{2} \operatorname{Tr}\left[F_{Q R} F_{M N}\right] \overline{\epsilon^{A}} \varepsilon_{P} Q R M N L_{L} \\
& +2 \partial_{M} \operatorname{Tr}\left[\overline{\lambda^{A}} \Gamma_{P} \lambda^{B}\right] \overline{\epsilon^{B}} \Gamma^{M}+2 \operatorname{Tr}\left[\overline{\lambda^{C}} \Gamma_{[P} D_{M]} \lambda^{C}\right] \overline{\epsilon^{A}} \Gamma^{M}
\end{aligned}
$$

where $\varepsilon_{012345}=1, T_{P M}$ is the stress tensor on shell given by

$$
T_{M P}=\frac{1}{4 g_{6}^{2}} \operatorname{Tr}\left[g_{M P} F_{Q N} F^{Q N}+4 F_{P}{ }^{N} F_{N M}-2 \overline{\lambda^{A}} \Gamma_{(M} D_{P)} \lambda^{A}\right] .
$$

A supercharge with $\operatorname{Sp}(1)$ index is defined by

$$
Q^{A}=\int d^{5} x S^{0 A}
$$

In the same argument given in section 2 , one can show that $\Delta \mathcal{O}=\left[-i \overline{\epsilon^{A}} Q^{A}, \mathcal{O}\right]$ for a gauge invariant operator $\mathcal{O}$. Then local form of supersymmetry algebra of SYM in six dimensions is determined as

$$
\left\{Q^{B}, \overline{S_{P}^{A}}\right\}=\left(-2 i \delta_{A}^{B} T_{P M}+\delta_{A}^{B} J_{P M}+\delta_{A}^{B} J_{P M}^{\prime}+J_{A}^{B} P M\right) \Gamma^{M}
$$

where

$$
\begin{aligned}
J_{P M} & =-\frac{i}{4 g_{6}^{2}} \operatorname{Tr}\left[F_{Q R} F_{L N}\right] \varepsilon_{P M}{ }^{Q R L N}, \\
J_{P M}^{\prime} & =-\frac{i}{2 g_{6}^{2}} \partial^{N} \operatorname{Tr}\left[\overline{\lambda^{C}} \Gamma_{P M N} \lambda^{C}\right], \\
J_{A P M}^{B} & =\frac{i}{g_{6}^{2}} \partial_{M} \operatorname{Tr}\left[\overline{\lambda^{A}} \Gamma_{P} \lambda^{B}\right] .
\end{aligned}
$$

There are several comments. Firstly as in ten dimensional case the contributions of fermions are given by total derivative terms. Secondly the terms in the right-hand side are all conserved, which is consistent with the fact that the SUSY current in the left-hand side is conserved. Especially for $J_{P M}, J_{P M}^{\prime}$, these are off-shell divergenceless. These antisymmetric tensors are not distinguishable in the algebra (3.17). There also exists non- $\mathrm{R}$ symmetric tensor $J_{A}^{B} P M$. Those tensors are so-called brane currents [32], which describes extended BPS objects in the theory.

One might ask whether total derivative terms of fermions appearing in the superalgebra are truly physical or not, since they may be absorbed by an improvement transformation preserving SUSY. ${ }^{4}$ A general study of this was done in four dimensions by using superfield formalism [32]. As a result an improvement transformation keeping SUSY including operators with spin not more than one was determined. ${ }^{5}$ And a general supercurrent multiplet called $\mathcal{S}$-multiplet was classified into several irreducible supercurrent multiplets by whether

\footnotetext{
${ }^{4}$ The author would like to thank the referee for raising this question.

${ }^{5}$ Existence of an improvement transformation to kill a total derivative term is not sufficient to decide the term as unphysical. To decide so, it also requires fields constructing the term to fall off fast enough at spatial infinity.
} 
there exists an improvement transformation to kill a submultiplet inside the $\mathcal{S}$-multiplet. To perform this kind of general analysis of supercurrent in the current case, it is important to develop superfield formalism in six dimensions which can determine an improvement transformation including higher spin operators. We leave these problems to future work.

Volume integration of both sides of (3.17) leads to supersymmetry algebra of six dimensional SYM theory as

$$
\left\{Q^{B}, \overline{Q^{A}}\right\}=\left(\delta_{A}^{B}\left(-2 i P_{M}+Z_{M}+Z_{M}^{\prime}\right)+Z_{A M}^{B}\right) \Gamma^{M}
$$

where we set

$$
P^{M}=\int d^{5} x T^{0 M}, \quad Z_{M}=\int d^{5} x J^{0}{ }_{M}, \quad Z_{M}^{\prime}=\int d^{5} x J^{\prime 0}{ }_{M}, \quad Z_{A M}^{B}=\int d^{5} x J_{A}^{B 0}{ }_{M} .
$$

$Z_{M}, Z_{M}^{\prime}, Z_{A M}^{B}$ are brane charges corresponding to the brane currents mentioned above.

\subsection{Inclusion of a hyper multiplet}

In this subsection we determine supersymmetry algebra of six dimensional SYM including a hyper multiplet. Extension to a multiple case is straightforward. A hyper multiplet consists of two complex scalar fields $q^{A}, A=1,2$, and a chiral fermion $\psi$ which has the opposite chirality to that of gaugino to interact therewith: $\hat{\Gamma} \psi=-\psi$. We consider a case where the hyper multiplet is in the fundamental representation of the gauge group for notational simplicity. Generalization to other representation can be easily done. The supersymmetric Lagrangian of the hyper multiplet is given by

$$
\mathcal{L}_{H}=-D_{M}\left(q^{A}\right)^{\dagger} D^{M} q^{A}+\frac{1}{2} \bar{\psi} \not D \psi+\varepsilon^{A B}\left(q^{A}\right)^{\dagger} \overline{\lambda^{B}} \psi-\varepsilon_{A B} \bar{\psi} \lambda^{A} q^{B}+\sqrt{2}\left(q^{A}\right)^{\dagger} D_{B}^{A} q^{B}
$$

and the supersymmetry transformation is determined as

$$
\begin{aligned}
\Delta q^{A} & =\varepsilon^{A B} \overline{\epsilon^{B}} \psi, & \Delta\left(q^{A}\right)^{\dagger} & =\varepsilon_{A B} \bar{\psi} \epsilon^{B}, \\
\Delta \psi & =2 \varepsilon_{B A} \Gamma^{M} \epsilon^{B} D_{M} q^{A}, & \Delta \bar{\psi} & =-2 \varepsilon^{B A} \bar{\epsilon}^{B} \Gamma^{M} D_{M}\left(q^{A}\right)^{\dagger} .
\end{aligned}
$$

The variation of the action of a hyper multiplet under the SUSY transformation is computed as

$$
\Delta\left(\int d^{6} x \mathcal{L}_{H}\right)=\int d^{6} x \overline{S_{M \text { hyp }}^{A}} \partial_{M} \epsilon^{A}
$$

where

$$
\overline{S_{P \text { hyp }}^{A}}=\varepsilon_{A B} \bar{\psi} \Gamma_{P} \Gamma^{N} D_{N} q^{B}-\left(D_{N} q^{A}\right)^{\dagger} \psi^{T} C_{6} \Gamma_{P} \Gamma^{N}-2\left(q^{A}\right)^{\dagger} \overline{\lambda^{B}} \Gamma_{P} q^{B}+\left(q^{B}\right)^{\dagger} \overline{\lambda^{A}} \Gamma_{P} q^{B} .
$$

Thus the supercurrent is given by

$$
\begin{aligned}
\overline{S_{P}^{A}}= & \frac{1}{2 g_{6}^{2}} \operatorname{Tr}\left[\overline{\lambda^{A}} F_{M N} \Gamma_{P} \Gamma^{M N}\right]+\varepsilon_{A B} \bar{\psi} \Gamma_{P} \Gamma^{N} D_{N} q^{B}-\left(D_{N} q^{A}\right)^{\dagger} \psi^{T} C_{6} \Gamma_{P} \Gamma^{N} \\
& -2\left(q^{A}\right)^{\dagger} \overline{\lambda^{B}} \Gamma_{P} q^{B}+\left(q^{B}\right)^{\dagger} \overline{\lambda^{A}} \Gamma_{P} q^{B} .
\end{aligned}
$$

Note that $S_{P}^{A}$ can be determined by using $\overline{\epsilon^{A}} S_{P}^{A}=\overline{S_{P}^{A}} \epsilon^{A}$. 
We can show that the supersymmetry current (3.28) is conserved: $\partial^{M} \overline{S_{M}^{A}}=0$ on shell. To show this, we need equations of motion of the gauge multiplet

$$
\begin{aligned}
\frac{1}{g_{6}^{2}} D_{N} F^{N M} & =-\frac{1}{g_{6}^{2}} \overline{\lambda^{A}} \Gamma^{M} \lambda^{A}+D^{M} q^{A}\left(q^{A}\right)^{\dagger}-q^{A} D^{M}\left(q^{A}\right)^{\dagger}-\frac{1}{2} \psi^{T}\left(\Gamma^{M}\right)^{T} \bar{\psi}^{T} \\
\frac{1}{g_{6}^{2}} D_{M} \overline{\lambda^{A}} \Gamma^{M} & =-\left(\varepsilon_{A B} q^{B} \bar{\psi}+\psi^{T} C_{6}\left(q^{A}\right)^{\dagger}\right) \\
D_{B}^{A} & =-\sqrt{2} g_{6}^{2}\left(q^{A}\left(q^{B}\right)^{\dagger}-\frac{1}{2} \delta_{B}^{A} q^{C}\left(q^{C}\right)^{\dagger}\right)
\end{aligned}
$$

and those of the hyper multiplet

$$
\begin{aligned}
& D^{2} q^{A}+\varepsilon^{A B} \overline{\lambda^{B}} \psi+\sqrt{2} D_{B}^{A} q^{B}=0, \\
& \frac{1}{2} \not D \psi+\varepsilon_{A B} \lambda^{B} q^{A}=0, \quad-\frac{1}{2} D_{M} \bar{\psi} \Gamma^{M}+\varepsilon^{A B}\left(q^{A}\right)^{\dagger} \overline{\lambda^{B}}=0 .
\end{aligned}
$$

We also need to employ another Fierz rearrangement

$$
\chi \bar{\psi}=\frac{-1}{4}\left(\bar{\psi} \chi+\frac{1}{2} \bar{\psi} \Gamma^{M N} \chi \Gamma_{N M}\right) \frac{1-(-)^{\psi} \hat{\Gamma}}{2}
$$

where $\psi, \chi$ are Weyl fermions with different chirality, and a formula

$$
\operatorname{Tr}\left[\overline{\lambda^{A}} \Gamma^{M}\left(\overline{\lambda^{B}} \Gamma_{M} \lambda^{B}\right)\right]=0 .
$$

Let us determine supersymmetry algebra in six dimensional SYM theory including a hyper multiplet. As seen from the equations of motion above, it is complicated to determine SUSY algebra including fermionic sector, thus we neglect the fermionic part in this paper, which we leave to future work. The variation of supercurrent under the supersymmetry transformation is computed as follows.

$$
\begin{aligned}
\Delta \overline{S_{P}^{A}}= & -2 T_{P M} \overline{\epsilon^{A}} \Gamma^{M}+\frac{1}{4 g_{6}^{2}} \operatorname{Tr}\left[F_{Q R} F_{M N}\right] \overline{\epsilon^{A}} \Gamma_{P} Q R M N \\
& -4 \partial_{M}\left[\left(q^{A}\right)^{\dagger} D_{N} q^{B}-\frac{1}{2} \delta_{A}^{B}\left(q^{C}\right)^{\dagger} D_{N} q^{C}\right] \overline{\epsilon^{B}} \Gamma_{P}{ }^{M N}
\end{aligned}
$$

where the stress tensor of the bosonic fields is given by

$$
\begin{aligned}
T_{M P}= & \frac{1}{4 g_{6}^{2}} \operatorname{Tr}\left[g_{M P}\left(F_{Q N} F^{Q N}+\frac{1}{2} D_{B}^{A} D_{A}^{B}\right)+4 F_{P}{ }^{N} F_{N M}\right] \\
& +2 D_{(M}\left(q^{A}\right)^{\dagger} D_{P)} q^{A}-g_{M P} \partial_{N}\left(\left(q^{A}\right)^{\dagger} D^{N} q^{A}\right) .
\end{aligned}
$$

Note that the quartic terms of the complex scalar fields vanish, which is required from consistency with conservation of the supercurrent in the left hand side of the superalgebra. As in the previous sections we can show that $\Delta \mathcal{O}=\left[-i \overline{\epsilon^{A}} Q^{A}, \mathcal{O}\right]$. Thus we obtain local form of supersymmetry algebra of six dimensional SYM including a hyper multiplet.

$$
\left\{Q^{B}, \overline{S_{P}^{A}}\right\}=\delta_{A}^{B}\left(-2 i T_{P M} \Gamma^{M}+J_{P M} \Gamma^{M}\right)+C_{A}^{B}{ }_{P Q R S} \Gamma^{S R Q}
$$


where

$$
C_{A}^{B P Q R S}=\frac{2 i}{3} \partial_{M}\left[\left(q^{A}\right)^{\dagger} D_{N} q^{B}-\frac{1}{2} \delta_{A}^{B}\left(q^{C}\right)^{\dagger} D_{N} q^{C}\right] \varepsilon^{P M N Q R S} .
$$

Thus supersymmetry algebra in six dimensional SYM including a hyper multiplet is obtained as

$$
\left\{Q^{B}, \overline{Q^{A}}\right\}=\delta_{A}^{B}\left(-2 i P_{M}+Z_{M}\right) \Gamma^{M}+Y_{A}^{B}{ }_{M N P} \Gamma^{P N M}
$$

where we set

$$
Y_{A}^{B Q R S}=\int d^{5} x C_{A}^{B 0 Q R S}
$$

\section{$3.36 \mathrm{~d} \mathcal{N}=2$ superalgebra}

In this section we determine supersymmetry algebra of $\mathcal{N}=2 \mathrm{SYM}$ by performing dimensional reduction from that of ten dimensional SYM, which was computed in section 2. $\mathcal{N}=$ $2 \mathrm{SYM}$ in six dimensions is constructed by a pair of vector multiplet and hyper multiplet in the adjoint representation. Thus six dimensional $\mathcal{N}=2 \mathrm{SYM}$ Lagrangian is given by addition of the Lagrangians of those multiplets, which were derived in the previous subsections.

$$
\begin{aligned}
\mathcal{L}^{\mathcal{N}=2}= & \frac{1}{g_{6}^{2}} \operatorname{Tr}\left[\frac{1}{4} F_{M N} F^{M N}+\frac{1}{2} \overline{\lambda^{A}} \not D \lambda^{A}+\frac{1}{2} D_{B}^{A} D^{B}{ }_{A}+\sqrt{2}\left(q^{A}\right)^{\dagger}\left[D^{A}{ }_{B}, q^{B}\right]\right. \\
& \left.-D_{M}\left(q^{A}\right)^{\dagger} D^{M} q^{A}+\frac{1}{2} \bar{\psi} \not D \psi+\varepsilon^{A B}\left(q^{A}\right)^{\dagger}\left[\overline{\lambda^{B}}, \psi\right]+\varepsilon_{A B} \bar{\psi}\left[\lambda^{B}, q^{A}\right]\right] .
\end{aligned}
$$

Note that the coupling constants of the vector multiplet and the hyper multiplet are the same. Integrating out the auxiliary field results in

$$
\begin{aligned}
\mathcal{L}^{\mathcal{N}=2}= & \frac{1}{g_{6}^{2}} \operatorname{Tr}\left[\frac{1}{4} F_{M N} F^{M N}+\frac{1}{2} \overline{\lambda^{A}} \not D \lambda^{A}-\frac{1}{2} D_{B}^{\prime A} D^{\prime B}{ }_{A}\right. \\
& \left.\quad-D_{M}\left(q^{A}\right)^{\dagger} D^{M} q^{A}+\frac{1}{2} \bar{\psi} \not D \psi+\varepsilon^{A B}\left(q^{A}\right)^{\dagger}\left[\overline{\lambda^{B}}, \psi\right]+\varepsilon_{A B} \bar{\psi}\left[\lambda^{B}, q^{A}\right]\right]
\end{aligned}
$$

where

$$
D_{B}^{\prime A}=-\sqrt{2}\left(\left[q^{A},\left(q^{B}\right)^{\dagger}\right]-\frac{1}{2} \delta_{B}^{A}\left[q^{C},\left(q^{C}\right)^{\dagger}\right]\right) .
$$

In order to determine $\mathcal{N}=2$ supersymmetry transformation and show that the Lagrangian (3.43) has sixteen maximal supersymmetry, we perform dimensional reduction for the SYM Lagrangian in ten dimensions. We compactify four directions $x^{m+5}$, where $m=1,2,3,4$. Then $X_{m}=-i A_{m+5}$ become four real scalar fields in six dimensions. We decompose the $\mathrm{SO}(1,9)$ gamma matrices denoted by $\Gamma_{M}^{(10)}$ as

$$
\Gamma_{M}^{(10)}=\Gamma_{M} \otimes \mathbf{1}, \quad \Gamma_{m+5}^{(10)}=\hat{\Gamma} \otimes \gamma_{m}
$$

where $\Gamma_{M}(M=0, \cdots, 5)$ are $\mathrm{SO}(1,5)$ gamma matrices and $\gamma_{m}$ are $\mathrm{SO}(4)$ gamma matrices, which we realize by a chiral expression

$$
\gamma_{m}=\left(\begin{array}{cc}
0 & \bar{\sigma}_{m} \\
\sigma_{m} & 0
\end{array}\right)
$$


where $\bar{\sigma}_{i}=\sigma_{i}(i=1,2,3)$ are Pauli matrices and $\bar{\sigma}_{4}=-\sigma_{4}=i$. Then the chirality matrix and charge conjugation matrix in ten dimensions are computed as

$$
\hat{\Gamma}_{10}=\hat{\Gamma} \otimes\left(\begin{array}{cc}
1 & 0 \\
0 & -1
\end{array}\right), \quad C_{10}=C_{6} \otimes\left(\begin{array}{cc}
i \sigma_{2} & 0 \\
0 & -i \sigma_{2}
\end{array}\right) \text {. }
$$

Therefore the Majorana-Weyl condition (2.1) in ten dimensions reduces to

$$
\lambda=\left(\begin{array}{c}
\lambda_{+}^{A} \\
\lambda_{-}^{A}
\end{array}\right), \quad \hat{\Gamma} \lambda_{ \pm}^{A}= \pm \lambda_{ \pm}^{A}, \quad \lambda_{ \pm}^{A}= \pm \varepsilon^{A B} C_{6}{\overline{\lambda_{ \pm}^{B}}}^{T},
$$

where $A=1,2$. This means that a ten-dimensional Majorana-Weyl fermion reduces to two sympletic-Majorana Weyl fermions $\lambda_{ \pm}^{A}$ in six dimensions. Then ten dimensional $\mathcal{N}=1$ SYM Lagrangian reduces to

$$
\begin{aligned}
& \mathcal{L}^{\mathcal{N}=2}=\frac{1}{g_{6}^{2}} \operatorname{Tr}\left[\frac{1}{4} F_{M N} F^{M N}-\frac{1}{2} D_{M} X_{m} D^{M} X^{m}+\frac{1}{4}\left[X_{m}, X_{n}\right]\left[X^{m}, X^{n}\right]\right. \\
& \left.+\frac{1}{2} \overline{\lambda_{+}^{A}} \Gamma^{M} D_{M} \lambda_{+}^{A}+\frac{1}{2} \overline{\lambda_{-}^{A}} \Gamma^{M} D_{M} \lambda_{-}^{A}+\frac{1}{2}\left(\overline{\lambda_{+}^{A}}\left(\bar{\sigma}_{m}\right)_{B}^{A}\left[i X^{m},-\lambda_{-}^{B}\right]+\overline{\lambda_{-}^{A}}\left(\sigma_{m}\right)_{B}^{A}\left[i X^{m}, \lambda_{+}^{B}\right]\right)\right] .
\end{aligned}
$$

Note that this Lagrangian has manifest $\mathrm{SO}(4) \simeq \mathrm{SU}(2) \times \mathrm{SU}(2)$ symmetry. This $\mathrm{SO}(4)$ symmetric Lagrangian (3.49) agrees with (3.43) under the following identification.

$$
\begin{array}{ll}
\lambda_{+}^{A}=\lambda^{A}, & \lambda_{-}^{1}=\frac{1}{\sqrt{2}} \psi, \\
X_{1}=\frac{\left(q^{1}\right)^{\dagger}-q^{1}}{\sqrt{2} i}, & X_{2}=\frac{\left(q^{1}\right)^{\dagger}+q^{1}}{-\sqrt{2}}, \quad X_{3}=\frac{q^{2}-\left(q^{2}\right)^{\dagger}}{\sqrt{2} i}, \quad X_{4}=\frac{q^{2}+\left(q^{2}\right)^{\dagger}}{\sqrt{2}} .
\end{array}
$$

The $\mathcal{N}=2$ supersymmetry transformation rule boils down to

$$
\begin{aligned}
\Delta A_{M} & =\overline{\epsilon_{+}^{A}} \Gamma_{M} \lambda_{+}^{A}+\overline{\epsilon_{-}^{A}} \Gamma_{M} \lambda_{-}^{A}, \\
\Delta X_{m} & \left.=i \overline{\epsilon_{+}^{A}}\left(\bar{\sigma}_{m}\right)^{A}{ }_{B} \lambda_{-}^{B}-\overline{\epsilon_{-}^{A}}\left(\sigma_{m}\right)^{A}{ }_{B} \lambda_{+}^{B}\right), \\
\Delta \lambda_{+}^{A} & =\frac{1}{2} F_{M N} \Gamma^{M N} \epsilon_{+}^{A}-i D_{M} X_{m} \Gamma^{M} \bar{\sigma}^{m A_{B}} \epsilon_{-}^{B}-\frac{1}{2}\left[X_{m}, X_{n}\right] \sigma^{m n A_{B} \epsilon_{+}^{B},} \\
\Delta \lambda_{-}^{A} & =\frac{1}{2} F_{M N} \Gamma^{M N} \epsilon_{-}^{A}+i D_{M} X_{m} \Gamma^{M} \sigma^{m A_{B}} \epsilon_{+}^{B}-\frac{1}{2}\left[X_{m}, X_{n}\right] \bar{\sigma}^{m n A_{B}} \epsilon_{-}^{B} .
\end{aligned}
$$

where the supersymmetry parameters $\epsilon_{ \pm}^{A}$ satisfy

$$
\hat{\Gamma} \epsilon_{ \pm}^{A}= \pm \epsilon_{ \pm}^{A}, \quad \epsilon_{ \pm}^{A}=\mp \varepsilon^{A B} C_{6} \bar{\epsilon}_{ \pm}^{B} .
$$

Therefore the type of SUSY is $(1,1)$.

Performing dimensional reduction for ten dimensional SUSY current (2.5), we obtain two supersymmetry currents in six dimensions.

$$
\begin{aligned}
& \overline{S_{-}^{A}}=\frac{1}{g_{6}^{2}} \operatorname{Tr}\left[\frac{1}{2} \overline{\lambda_{+}^{A}} F_{M N} \Gamma^{P} \Gamma^{M N}-i \overline{\lambda_{-}^{B}}\left(\bar{\sigma}^{n}\right)^{B}{ }_{A} \Gamma^{P} \Gamma^{M} D_{M} X_{n}-\frac{1}{2} \overline{\lambda_{+}^{B}}\left(\sigma^{m n}\right)^{B}{ }_{A}\left[X_{m}, X_{n}\right] \Gamma^{P}\right], \\
& \overline{S_{+}{ }_{P}^{A}}=\frac{1}{g_{6}^{2}} \operatorname{Tr}\left[\frac{1}{2} \overline{\lambda_{-}^{A}} F_{M N} \Gamma^{P} \Gamma^{M N}+i \overline{\lambda_{+}^{B}}\left(\sigma^{n}\right)^{B}{ }_{A} D_{M} X_{n} \Gamma^{P} \Gamma^{M}-\frac{1}{2} \overline{\lambda_{-}^{B}}\left(\bar{\sigma}^{m n}\right)^{B}{ }_{A}\left[X_{m}, X_{n}\right] \Gamma^{P}\right] .
\end{aligned}
$$


The $\mathcal{N}=2$ supersymmetry algebra in six dimensions is computed by dimensional reduction of that of ten dimensional $\mathcal{N}=1$ algebra calculated in section 2 . To simplify the situation, we ignore the contributions of fermions. SUSY charges are

$$
Q_{ \pm}^{A}=\int d^{5} x S_{ \pm}^{0 A}
$$

and $\Delta \mathcal{O}=-i\left[\overline{\epsilon_{+}^{B}} Q_{-}^{B}+\overline{\epsilon_{-}^{B}} Q_{+}^{B}, \mathcal{O}\right]$, which is justified by the ten dimensional result. From (2.20) we calculate the local form of SUSY algebra of $\mathcal{N}=2$ SYM in six dimensions.

$$
\begin{aligned}
& \left\{Q_{-}^{B}, \overline{S_{-}^{A}}\right\}=-2 i \delta^{B}{ }_{A} T_{P M} \Gamma^{M}+J_{P M} \delta^{B}{ }_{A} \Gamma^{M}+C^{n m}{ }_{P}{ }^{Q R S} \sigma^{n m B}{ }_{A} \Gamma_{S R Q}, \\
& \left\{Q_{+}^{B}, \overline{S_{-} A}\right\}=-2 i T_{P m} \sigma^{m B}{ }_{A}+C_{P K L}^{m} \sigma_{m}{ }^{B}{ }_{A} \Gamma^{L K}+C^{l}{ }_{P}{ }^{Q R S T} \sigma_{l}{ }_{A}{ }_{A} \Gamma_{T S R Q}, \\
& \left\{Q_{+}^{B}, \overline{S_{+}{ }_{P}}\right\}=-2 i \delta^{B}{ }_{A} T_{P M} \Gamma^{M}-J_{P M} \delta^{B}{ }_{A} \Gamma^{M}-C^{n m}{ }_{P}{ }^{Q R S} \bar{\sigma}^{n m B}{ }_{A} \Gamma_{S R Q}, \\
& \left\{Q_{-}^{B}, \overline{S_{-}{ }_{P}^{A}}\right\}=2 i T_{P m} \bar{\sigma}^{m B}{ }_{A}+C_{P K L}^{m} \bar{\sigma}_{m}{ }^{B}{ }_{A} \Gamma^{L K}-C_{P}^{l}{ }_{P}{ }^{Q R S T} \bar{\sigma}_{l}{ }^{B}{ }_{A} \Gamma_{T S R Q},
\end{aligned}
$$

where we used the equation of motion of the gauge field

$$
D_{M} F_{P}^{M}-\left[D_{P} X^{n}, X_{n}\right]=0,
$$

and we set

$$
\begin{aligned}
T_{P M}= & \frac{-1}{4 g_{6}^{2}} \operatorname{Tr}\left[g_{M P}\left(F_{N Q} F^{N Q}+2 D_{M} X_{m} D^{P} X^{m}-\left[X_{m}, X_{n}\right]\left[X^{m}, X^{n}\right]\right)\right. \\
& \left.+4\left(F_{M}^{N} F_{N P}-D_{M} X^{m} D_{P} X_{m}\right)\right] \\
T_{P m}= & \frac{i}{g_{6}^{2}} \partial_{N} \operatorname{Tr}\left[F_{P}^{N} X_{m}\right] \\
C_{P}^{n m}{ }_{P}^{Q R S}= & \frac{-i}{6 g_{6}^{2}} \varepsilon_{P}^{M N Q R S} \partial_{M} \operatorname{Tr}\left[X^{n} D_{N} X^{m}\right] \\
C_{P K L}^{m}= & \frac{-1}{4 g_{6}^{2}} \varepsilon_{P R M N K L} \partial^{R} \operatorname{Tr}\left[X^{m} F^{M N}\right] \\
C_{l P} Q R S T= & \frac{-1}{72 g_{6}^{2}} \varepsilon_{P}^{N Q R S T} \sigma_{q r m l} \partial_{N} \operatorname{Tr}\left[\left[X^{q}, X^{r}\right], X^{m}\right] .
\end{aligned}
$$

Note that $\sigma_{1234}=1$. This leads to supersymmetry algebra of six dimensional $\mathcal{N}=2 \mathrm{SYM}$.

$$
\begin{aligned}
& \left\{Q_{-}^{B}, \overline{Q_{-}^{A}}\right\}=\left(-2 i P_{M}+Z_{M}\right) \Gamma^{M} \delta_{A}^{B}+Z_{Q R S}^{m n} \sigma_{m n}{ }^{B}{ }_{A} \Gamma^{S R Q}, \\
& \left\{Q_{+}^{B}, \overline{Q_{-}^{A}}\right\}=-2 i P_{m} \sigma^{m B}{ }_{A}+Z_{M N}^{m} \sigma_{m}{ }^{B}{ }_{A} \Gamma^{N M}+Z_{Q R S T}^{m} \sigma_{m}{ }_{A}{ }_{A} \Gamma^{T S R Q}, \\
& \left\{Q_{+}^{B}, \overline{Q_{+}^{A}}\right\}=\left(-2 i P_{M}-Z_{M}\right) \Gamma^{M} \delta_{A}^{B}-Z_{Q R S}^{m n} \bar{\sigma}_{m n}{ }^{B}{ }_{A} \Gamma^{S R Q} \\
& \left\{Q_{-}^{B}, \overline{Q_{+}^{A}}\right\}=2 i P_{m} \bar{\sigma}^{m B}{ }_{A}+Z_{M N}^{m} \bar{\sigma}_{m}{ }^{B}{ }_{A} \Gamma^{N M}-Z_{Q R S T}^{m} \bar{\sigma}_{m}{ }^{B}{ }_{A} \Gamma^{T S R Q},
\end{aligned}
$$

where

$$
\begin{aligned}
& P^{M}=\int d^{5} x T^{0 M}, \quad P^{m}=\int d^{5} x T^{0 m}, \\
& Z_{M N P}^{m n}=\int d^{5} x C^{m n 0}{ }_{M N P}, \quad Z_{K L}^{m}=\int d^{5} x C^{m 0}{ }_{K L}, \quad Z_{Q R S T}^{l}=\int d^{5} x C^{l 0}{ }_{Q R S T} .
\end{aligned}
$$




\section{Superalgebra in 5d SYM}

In this section we study supersymmetry algebra of SYM in five dimensions. We derive this by dimensional reduction from higher dimensional SUSY algebra studied in the previous sections.

\subsection{Vector multiplet}

We first consider SYM consisting of one vector multiplet. This theory can be obtained by dimensional reduction of six dimensional theory studied in section 3.1. We degenerate the fifth direction and the gauge field of this direction becomes a scalar field in the adjoint representation, $A_{5}=i \varphi$, where $\varphi$ takes a real value. We decompose the gamma matrices in six dimensions into five dimensions ones in the following way.

$$
\Gamma_{\mu}=\gamma_{\mu} \otimes \sigma_{1}, \quad \Gamma_{5}=\mathbf{1} \otimes \sigma_{2} .
$$

Then the six dimensional chirality matrix and the charge conjugation matrix is computed as

$$
\hat{\Gamma}=\mathbf{1} \otimes \sigma_{3}, \quad C_{6}=C_{5} \otimes i \sigma_{2},
$$

where $C_{5}=-i \gamma_{03}$. The $\operatorname{Sp}(1)$-Majorana Weyl fermion $\lambda_{6 d}^{A}$ in six dimension constrained by (3.1) reduces to

$$
\lambda_{6 d}^{A}=\left(\begin{array}{c}
\lambda^{A} \\
\lambda^{\prime A}
\end{array}\right), \quad \lambda^{\prime A}=0, \quad \varepsilon^{A B} C_{5} \overline{\lambda^{B}}=\lambda^{A},
$$

which is simply the $\mathrm{Sp}(1)$-Majorana condition in five dimensions.

From the six dimensional Lagrangian of $\mathcal{N}=1 \mathrm{SYM}$ of a vector multiplet given by (3.3), we obtain that of five dimensional one as

$$
\mathcal{L}_{V}=\frac{1}{g_{5}^{2}} \operatorname{Tr}\left[\frac{1}{4} F_{\mu \nu} F^{\mu \nu}-\frac{1}{2} D_{\mu} \varphi D^{\mu} \varphi+\frac{1}{2} D^{A}{ }_{B} D^{B}{ }_{A}+\frac{1}{2} \overline{\lambda^{A}} \not D \lambda^{A}-\frac{1}{2} \overline{\lambda^{A}}\left[\varphi, \lambda^{A}\right]\right]
$$

where $g_{5}$ is a coupling constant of this theory and $\not D=\gamma^{\mu} D_{\mu}$. The SUSY transformation rule can also be obtained from six dimensional one given by (3.4).

$$
\begin{aligned}
\Delta A_{\mu} & =\overline{\epsilon^{A}} \gamma_{\mu} \lambda^{A}, \\
\Delta \varphi & =\overline{\epsilon^{A}} \lambda^{A}, \\
\Delta \lambda^{A} & =\frac{1}{2} F_{\mu \nu} \gamma^{\mu \nu} \epsilon^{A}-\not D \varphi \epsilon^{A}+\alpha D_{B}^{A} \epsilon^{B}, \\
\Delta D_{B}^{A} & =\alpha\left(D_{\mu} \overline{\lambda^{B}} \gamma^{\mu} \epsilon^{A}-\left[\varphi, \overline{\lambda^{B}}\right] \epsilon^{A}-\frac{1}{2} \delta_{B}^{A}\left(D_{\mu} \overline{\lambda^{C}} \gamma^{\mu} \epsilon^{C}-\left[\varphi, \overline{\lambda^{C}}\right] \epsilon^{C}\right)\right),
\end{aligned}
$$

where $\epsilon^{A}$ is a fermionic supersymmetry parameter satisfying $-\varepsilon^{A B} C_{5} \overline{\epsilon^{B}}=\epsilon^{A} . \alpha$ is arbitrary when we consider only a vector multiplet while $\alpha=\sqrt{2}$ when a hyper multiplet is introduced. The supersymmetry current of the Lagrangian (4.4) is computed by dimensional reduction from (3.5).

$$
\overline{S_{\rho}^{A}}=\frac{1}{g_{5}^{2}} \operatorname{Tr}\left[\overline{\lambda^{A}}\left(\frac{1}{2} F_{\mu \nu} \gamma^{\rho} \gamma^{\mu \nu}-D_{\mu} \varphi \gamma^{\rho} \gamma^{\mu}\right)\right] .
$$


The supersymmtery algebra of this theory can be computed in the same way. The supersymmetry variation of the SUSY current is computed as follows.

$$
\begin{aligned}
\Delta \overline{S_{\rho}^{A}}= & -2 T_{\rho \mu} \overline{\epsilon^{A}} \gamma^{\mu}+2 i T_{\rho 5} \overline{\epsilon^{A}} \\
& +\frac{1}{4 g_{5}^{2}}\left(\operatorname{Tr}\left[F_{\mu \nu} F_{\sigma \lambda}\right] \overline{\epsilon^{A}} \gamma_{\rho}^{\mu \nu \sigma \lambda}+4 \partial_{\sigma} \operatorname{Tr}\left[F_{\mu \nu} \varphi\right] \overline{\epsilon^{A}} \gamma_{\rho}^{\mu \nu \sigma \lambda} \gamma_{\lambda}+2 \partial_{\mu} \operatorname{Tr}\left[\varphi D_{\sigma} \varphi\right] \overline{\epsilon^{A}} \gamma_{\rho}^{\mu \sigma \nu \lambda} \gamma_{\lambda \nu}\right) \\
& +\frac{1}{g_{5}^{2}} \partial_{\mu} \operatorname{Tr}\left[\overline{\lambda^{A}} \gamma_{\rho} \lambda^{B}\right] \overline{\epsilon^{B}} \gamma^{\mu}-\frac{1}{2 g_{5}^{2}} \partial^{\nu}\left(\operatorname{Tr}\left[\overline{\lambda^{C}} \gamma_{\rho \mu \nu} \lambda^{C}\right] \overline{\epsilon^{A}} \gamma^{\mu}+\operatorname{Tr}\left[\overline{\lambda^{C}}\left(-\gamma_{\rho \nu}\right) \lambda^{C}\right] \overline{\epsilon^{A}}\right)
\end{aligned}
$$

where $\gamma_{01234}=-i$,

$$
\begin{aligned}
& T_{\mu \rho}=\frac{1}{4 g_{5}^{2}} \operatorname{Tr}\left[g_{\mu \rho}\left(F_{\nu \sigma} F^{\nu \sigma}-2 D_{\nu} \varphi D^{\nu} \varphi\right)+4\left(F_{\mu}{ }^{\nu} F_{\nu \rho}+D_{\mu} \varphi D_{\rho} \varphi\right)-2 \overline{\lambda^{A}} \gamma_{(\mu} D_{\rho)} \lambda^{A}\right], \\
& T_{\rho 5}=\frac{i}{g_{5}^{2}} \partial_{\nu} \operatorname{Tr}\left[F_{\rho}{ }^{\nu} \varphi\right]+\frac{1}{4 g_{5}^{2}} \operatorname{Tr}\left[-i \overline{\lambda^{A}} \gamma_{\rho}\left[\varphi, \lambda^{A}\right]-i \overline{\lambda^{A}} D_{\rho} \lambda^{A}\right] .
\end{aligned}
$$

The supercharge is

$$
Q^{A}=\int d^{4} x S^{0 A}
$$

and SUSY transformation is given by $\Delta \mathcal{O}=\left[-i \overline{\epsilon^{A}} Q^{A}, \mathcal{O}\right]$, which is already justified from the higher dimensional result. Thus anti-commutation relation of the supercharge and supercurrent is computed as

$$
\left\{Q^{B}, \overline{S^{\rho A}}\right\}=\delta_{A}^{B}\left(-2 i T_{\rho \mu} \gamma^{\mu}-2 T_{\rho 5}+j_{\rho}+j_{\rho}^{\prime}+\left(j_{\rho \mu}+j_{\rho \mu}^{\prime}\right) \gamma^{\mu}+j_{\rho \nu \lambda} \gamma^{\lambda \nu}\right)+j_{A}^{\prime B}{ }_{\rho \mu} \gamma^{\mu}
$$

where we set

$$
\begin{aligned}
j_{\rho} & =\frac{i}{4 g_{5}^{2}} \operatorname{Tr}\left[F_{\mu \nu} F_{\sigma \lambda}\right] \gamma_{\rho}^{\mu \nu \sigma \lambda}, \\
j_{\rho \lambda} & =\frac{i}{g_{5}^{2}} \partial_{\sigma} \operatorname{Tr}\left[F_{\mu \nu} \varphi\right] \gamma_{\rho}^{\mu \nu \sigma}{ }_{\lambda}, \\
j_{\rho \nu \lambda} & =\frac{i}{2 g_{5}^{2}} \partial_{\mu} \operatorname{Tr}\left[\varphi D_{\sigma} \varphi\right] \gamma_{\rho}^{\mu \sigma}{ }_{\nu \lambda}, \\
j_{A \rho \mu}^{B B} & =\frac{i}{g_{5}^{2}} \partial_{\mu} \operatorname{Tr}\left[\overline{\lambda^{A}} \gamma_{\rho} \lambda^{B}\right], \\
j_{\rho \mu}^{\prime} & =-\frac{i}{2 g_{5}^{2}} \partial^{\nu}\left(\operatorname{Tr}\left[\overline{\lambda^{C}} \gamma_{\rho \mu \nu} \lambda^{C}\right],\right. \\
j_{\rho}^{\prime} & =\frac{i}{2 g_{5}^{2}} \partial^{\nu} \operatorname{Tr}\left[\overline{\lambda^{C}} \gamma_{\rho \nu} \lambda^{C}\right] .
\end{aligned}
$$

Note that $j_{\rho}$ is instanton-particle number current. Volume integration of both sides gives SUSY algebra of five dimensional SYM as

$$
\left\{Q^{B}, \overline{Q^{A}}\right\}=\delta_{A}^{B}\left(\left(-2 i P_{\mu}+Z_{\mu}+Z_{\mu}^{\prime}\right) \gamma^{\mu}-2 P_{5}+Z+Z^{\prime}+Z_{\nu \lambda} \gamma^{\lambda \nu}\right)+Z_{A \mu}^{\prime B} \gamma^{\mu}
$$

where

$$
P^{\mu}=\int d^{4} x T^{0 \mu}, \quad P^{5}=\int d^{4} x T^{05}
$$




$$
\begin{aligned}
& Z=\int d^{4} x j^{0}, \quad Z_{\lambda}=\int d^{4} x j^{0}{ }_{\lambda}, \quad Z_{\nu \lambda}=\int d^{4} x j^{0}{ }_{\nu \lambda}, \\
& Z_{A \mu}^{\prime B}=\int d^{4} x j_{A}^{\prime B 0}{ }_{\mu}, \quad Z_{\mu}^{\prime}=\int d^{4} x j^{\prime 0}{ }_{\mu}, \quad Z^{\prime}=\int d^{4} x j^{\prime 0} .
\end{aligned}
$$

$P^{5}$ is the Kaluza-Klein momentum arising by circle compactification of six dimensional SYM and $Z$ is the instanton-particle charge. These are different but indistinguishable in the superalgebra.

\subsection{Inclusion of a hyper multiplet}

We can obtain a theory of a hyper multiplet in five dimensions by dimensional reduction for six dimensional theory studied in section 3.2. The chiral fermion in six dimensions denoted by $\psi_{6 d}$ reduces to

$$
\psi_{6 d}=\left(\begin{array}{c}
\psi^{\prime} \\
\psi
\end{array}\right), \quad \psi^{\prime}=0 .
$$

By using this notation we obtain the Lagrangian of a hyper multiplet from (3.23).

$$
\begin{aligned}
\mathcal{L}_{H}= & -D_{M}\left(q^{A}\right)^{\dagger} D^{M} q^{A}-\left(q^{A}\right)^{\dagger} \varphi^{2} q^{A}+\frac{1}{2} \bar{\psi} \not D \psi+\frac{1}{2} \bar{\psi} \varphi \psi \\
& +\varepsilon^{A B}\left(q^{A}\right)^{\dagger} \overline{\lambda^{B}} \psi-\varepsilon_{A B} \bar{\psi} \lambda^{A} q^{B}+\sqrt{2}\left(q^{A}\right)^{\dagger} D^{A}{ }_{B} q^{B} .
\end{aligned}
$$

The SUSY transformation is computed from six dimensional one as

$$
\Delta q^{A}=\varepsilon^{A B} \overline{\epsilon^{B}} \psi, \quad \Delta \psi=2 \varepsilon_{B A}\left(\gamma^{\mu} \epsilon^{B} D_{\mu}-\epsilon^{B} \varphi\right) q^{A} .
$$

Five dimensional SUSY current is computed as

$$
\begin{aligned}
\overline{S_{\rho}^{A}}= & \frac{1}{g_{5}^{2}} \operatorname{Tr}\left[\overline{\lambda^{A}}\left(\frac{1}{2} F_{\mu \nu} \gamma^{\rho} \gamma^{\mu \nu}-D_{\mu} \varphi \gamma^{\rho} \gamma^{\mu}\right)\right]+\varepsilon_{A B} \overline{\psi_{-}} \gamma_{\rho}\left(\gamma^{\nu} D_{\nu} q^{B}-\varphi q^{B}\right) \\
& +\left(D_{\nu} q^{A}\right)^{\dagger} \psi^{T} C_{5} \gamma_{\rho} \gamma^{\nu}-\left(q^{A}\right)^{\dagger} \varphi \psi^{T} C_{5} \gamma_{\rho}-2\left(q^{A}\right)^{\dagger} \overline{\lambda^{B}} \gamma_{\rho} q^{B}+\left(q^{B}\right)^{\dagger} \overline{\lambda^{A}} \gamma_{\rho} q^{B} .
\end{aligned}
$$

Supersymmetry variation of supercurrent is computed from (3.36) as

$$
\begin{aligned}
\Delta \overline{S_{\rho}^{A}}= & -2 T_{\rho \mu} \overline{\epsilon^{A}} \gamma^{\mu}+2 i T_{\rho 5} \overline{\epsilon^{A}} \\
& +\frac{1}{4 g_{5}^{2}}\left(\operatorname{Tr}\left[F_{\mu \nu} F_{\sigma \lambda}\right] \overline{\epsilon^{A}} \gamma_{\rho}^{\mu \nu \sigma \lambda}+4 \partial_{\sigma} \operatorname{Tr}\left[F_{\mu \nu} \varphi\right] \overline{\epsilon^{A}} \gamma_{\rho}^{\mu \nu \sigma \lambda} \gamma_{\lambda}+2 \partial_{\mu} \operatorname{Tr}\left[\varphi D_{\sigma} \varphi\right] \overline{\epsilon^{A}} \gamma_{\rho}^{\mu \sigma \nu \lambda} \gamma_{\lambda \nu}\right) \\
& -2 \partial_{\mu}\left[\left(q^{A}\right)^{\dagger} D_{\nu} q^{B}-\frac{1}{2} \delta_{A}^{B}\left(q^{C}\right)^{\dagger} D_{\nu} q^{C}\right] \overline{\epsilon^{B}} \gamma_{\rho}^{\mu \nu \sigma \lambda} \gamma_{\lambda \sigma} \\
& +4 \partial_{\mu}\left[\left(q^{A}\right)^{\dagger} \varphi q^{B}-\frac{1}{2} \delta_{A}^{B}\left(q^{C}\right)^{\dagger} \varphi q^{C}\right] \overline{\epsilon^{B}} \gamma_{\rho}{ }^{\mu}
\end{aligned}
$$

where the stress tensor of the bosonic fields is given by

$$
\begin{aligned}
T_{\mu \rho}= & \frac{1}{4 g_{5}^{2}} \operatorname{Tr}\left[g_{\mu \rho}\left(F_{\nu \sigma} F^{\nu \sigma}-2 D_{\nu} \varphi D^{\nu} \varphi\right)+4\left(F_{\mu}{ }^{\nu} F_{\nu \rho}+D_{\mu} \varphi D_{\rho} \varphi\right)\right] \\
& +2 D_{(\mu}\left(q^{A}\right)^{\dagger} D_{\rho)} q^{A}-g_{\mu \rho} \partial_{\nu}\left(\left(q^{A}\right)^{\dagger} D^{\nu} q^{A}\right)
\end{aligned}
$$




$$
T_{\rho 5}=\frac{i}{g_{5}^{2}} \partial_{\nu} \operatorname{Tr}\left[F_{\rho}{ }^{\nu} \varphi\right]+i D_{\mu}\left(q^{A}\right)^{\dagger} \varphi q^{A}-i\left(q^{A}\right)^{\dagger} \varphi D_{\mu} q^{A}
$$

By using $\Delta \mathcal{O}=\left[-i \overline{\epsilon^{A}} Q^{A}, \mathcal{O}\right]$, we obtain local supersymmetry algebra of five dimensional SYM including a hyper multiplet.

$$
\left\{Q^{B}, \overline{S_{\rho}^{A}}\right\}=\delta_{A}^{B}\left(-2 i T_{\rho \mu} \gamma^{\mu}-2 T_{\rho 5}+j_{\rho}+j_{\rho \lambda} \gamma^{\lambda}+j_{\rho \nu \lambda} \gamma^{\lambda \nu}\right)+c_{A \rho \sigma \lambda}^{B} \gamma^{\lambda \sigma}+c_{A \rho \nu \sigma \lambda}^{B} \gamma^{\lambda \sigma \nu}
$$

where

$$
\begin{aligned}
c_{A \rho \sigma \lambda}^{B} & =-2 i \partial_{\mu}\left[\left(q^{A}\right)^{\dagger} D_{\nu} q^{B}-\frac{1}{2} \delta_{A}^{B}\left(q^{C}\right)^{\dagger} D_{\nu} q^{C}\right] \gamma_{\rho}^{\mu \nu} \sigma \lambda, \\
c_{A \rho}^{B}{ }^{\nu \sigma \lambda} & =\frac{2 i}{3} \partial_{\mu}\left[\left(q^{A}\right)^{\dagger} \varphi q^{B}-\frac{1}{2} \delta_{A}^{B}\left(q^{C}\right)^{\dagger} \varphi q^{C}\right] \gamma_{\rho}^{\mu \nu \sigma \lambda} .
\end{aligned}
$$

Thus supersymmetry algebra in SYM in five dimensions is obtained as

$$
\left\{Q^{B}, \overline{Q^{A}}\right\}=\delta_{A}^{B}\left(\left(-2 i P_{\mu}+Z_{\mu}\right) \gamma^{\mu}-2 P_{5}+Z+Z_{\nu \lambda} \gamma^{\lambda \nu}\right)+Y_{A \mu \nu \sigma}^{B} \gamma^{\sigma \nu \mu}+Y_{A \nu \mu}^{B} \gamma^{\mu \nu}
$$

where $Z, Z_{\mu}, Z_{\nu \lambda}$ are given by (4.19), and

$$
P^{\mu}=\int d^{4} x T^{0 \mu}, \quad P^{5}=\int d^{4} x T^{05}, \quad Y_{A}^{B \nu \mu}=\int d^{4} x c_{A}^{B 0 \nu \mu}, \quad Y_{A}^{B \nu \sigma \lambda}=\int d^{4} x c_{A}^{B 0 \nu \sigma \lambda} .
$$

Note that one can add a real mass parameter $m$ for the hyper multiplet by giving a vev to the adjoint scalar field $\varphi$. Modification of SUSY algebra is done by replacing $\varphi \rightarrow m+\varphi$.

\section{$4.35 \mathrm{~d} \mathcal{N}=2$ superalgebra}

We study SUSY algebra of maximally supersymmetric Yang-Mills theory in five dimensions in this subsection. This was studied in a different notation in [18]. This theory can be obtained by reducing six dimensional $\mathcal{N}=2 \mathrm{SYM}$ to five dimensional one. Two $\operatorname{Sp}(1)-$ Majorana Weyl fermions in six dimensions denoted by $\lambda_{ \pm}^{A}$ in section 3.3 reduces to

$$
\lambda_{+}^{A}=\left(\begin{array}{c}
\lambda_{1}^{A} \\
\lambda_{1}^{\prime A}
\end{array}\right), \quad \lambda_{-}^{A}=\left(\begin{array}{c}
\lambda_{2}^{\prime A} \\
\lambda_{2}^{A}
\end{array}\right)
$$

with

$$
\lambda_{1}^{\prime A}=\lambda_{2}^{\prime A}=0, \quad \varepsilon^{A B} C_{5}{\overline{\lambda_{1}^{B}}}^{T}=\lambda_{1}^{A}, \quad \varepsilon^{A B} C_{5} \overline{\lambda_{2}^{B}}=\lambda_{2}^{A} .
$$

The six dimensional $\mathcal{N}=2$ Lagrangian given by (3.49) reduces to

$$
\begin{aligned}
\mathcal{L}^{\mathcal{N}=2}= & \frac{1}{g_{5}^{2}} \operatorname{Tr}\left[\frac{1}{4} F_{\mu \nu} F^{\mu \nu}-\frac{1}{2} D_{\mu} \varphi D^{\mu} \varphi-\frac{1}{2} D_{\mu} X_{m} D^{\mu} X^{m}+\frac{1}{2}\left[\varphi, X_{m}\right]\left[\varphi, X^{m}\right]+\frac{1}{4}\left[X_{m}, X_{n}\right]\left[X^{m}, X^{n}\right]\right. \\
& +\frac{1}{2} \overline{\lambda_{1}^{A}} \gamma^{\mu} D_{\mu} \lambda_{1}^{A}-\frac{1}{2} \overline{\lambda_{1}^{A}}\left[\varphi, \lambda_{1}^{A}\right]+\frac{1}{2} \overline{\lambda_{2}^{A}} \gamma^{\mu} D_{\mu} \lambda_{2}^{A}+\frac{1}{2} \overline{\lambda_{2}^{A}}\left[\varphi, \lambda_{2}^{A}\right] \\
& \left.+\frac{1}{2}\left(-i \overline{\lambda_{1}^{A}}\left(\bar{\sigma}_{m}\right)_{B}^{A}\left[X^{m}, \lambda_{2}^{B}\right]+i \overline{\lambda_{2}^{A}}\left(\sigma_{m}\right)_{B}^{A}\left[X^{m}, \lambda_{1}^{B}\right]\right)\right] .
\end{aligned}
$$

Five dimensional $\mathcal{N}=2$ transformation rule is obtained from (3.51).

$$
\Delta A_{\mu}=\overline{\epsilon_{1}^{A}} \gamma_{\mu} \lambda_{1}^{A}+\overline{\epsilon_{2}^{A}} \gamma_{\mu} \lambda_{2}^{A},
$$




$$
\begin{aligned}
& \Delta \varphi=\overline{\epsilon_{1}^{A}} \lambda_{1}^{A}-\overline{\epsilon_{2}^{A}} \lambda_{2}^{A} \\
& \Delta X_{m}=i \overline{\epsilon_{1}^{A}}\left(\bar{\sigma}_{m}\right)^{A}{ }_{B} \lambda_{2}^{B}-i \overline{\epsilon_{2}^{A}}\left(\sigma_{m}\right)^{A}{ }_{B} \lambda_{1}^{B}, \\
& \Delta \lambda_{1}^{A}=\frac{1}{2} F_{\mu \nu} \gamma^{\mu \nu} \epsilon_{1}^{A}-\not D \varphi \epsilon_{1}^{A}-i \not D X_{m} \bar{\sigma}^{m A_{B}} \epsilon_{2}^{B}-i\left[\varphi, X_{m}\right] \bar{\sigma}^{m A_{B}} \epsilon_{2}^{B}-\frac{1}{2}\left[X_{m}, X_{n}\right] \sigma^{m n A_{B}} \epsilon_{1}^{B} \text {, } \\
& \Delta \lambda_{2}^{A}=\frac{1}{2} F_{\mu \nu} \gamma^{\mu \nu} \epsilon_{2}^{A}+\not D \varphi \epsilon_{2}^{A}+i \not D X_{m} \sigma^{m A_{B}} \epsilon_{1}^{B}-i\left[\varphi, X_{m}\right] \sigma_{B}^{m A_{B}} \epsilon_{1}^{B}-\frac{1}{2}\left[X_{m}, X_{n}\right] \bar{\sigma}^{m n A_{B}} \epsilon_{2}^{B} \text {, }
\end{aligned}
$$

where $\epsilon_{1}, \epsilon_{2}$ are fermionic SUSY parameters satisfying $\varepsilon^{A B} C_{5}{\overline{\epsilon_{1}^{B}}}^{T}=-\epsilon_{1}^{A}, \varepsilon^{A B} C_{5} \overline{\epsilon_{2}^{B}}=-\epsilon_{2}^{A}$

One can rewrite the Lagrangian (4.35) in a $\mathrm{SO}(5)$ symmetric form. This can be easily done by dimensional reduction from ten dimensional SYM. Degenerating the five directions $x^{I}$, where $I=5,6,7,8,9$, we obtain five real scalar fields from the gauge fields of those directions, denoting by $X_{I}=-i A_{I}$. We decompose the ten dimensional gamma matrices in a way that

$$
\Gamma_{\mu}=\gamma_{\mu} \otimes \mathbf{1} \otimes \sigma_{1}, \quad \Gamma_{I}=\mathbf{1} \otimes \gamma_{I} \otimes \sigma_{2}
$$

where $\gamma_{\mu}(\mu=0, \cdots, 4)$ are $\mathrm{SO}(1,4)$ gamma matrices, $\gamma_{I}$ are $\mathrm{SO}(5)$ gamma matrices. Then ten dimensional chirality matrix and charge conjugation matrix are computed as

$$
\hat{\Gamma}_{10}=\mathbf{1} \otimes \mathbf{1} \otimes \sigma_{3}, \quad C_{10}=C_{5} \otimes \omega \otimes-i \sigma_{2}
$$

where $\omega=\gamma_{68}$ is an $\operatorname{Sp}(2)$ invariant matrix. The Majorana-Weyl condition in ten dimensions (2.1) reduces to

$$
\lambda=\left(\begin{array}{c}
\lambda^{\mathbf{A}} \\
\lambda^{\prime \mathbf{A}}
\end{array}\right), \quad \lambda^{\prime \mathbf{A}}=0, \quad \lambda^{\mathbf{A}}=\omega^{\mathbf{A B}} C_{5}{\overline{\lambda^{\mathbf{B}}}}^{T},
$$

where $\mathbf{A}=1,2,3,4$. In other words, a ten dimensional Majorana-Weyl fermion reduces to an $\mathrm{Sp}(2)$-Majorana fermion in five dimensions. Under this notation the ten dimensional SYM Lagrangian reduces to

$$
\begin{aligned}
\mathcal{L}^{\mathcal{N}=2}= & \frac{1}{g_{5}^{2}} \operatorname{Tr}\left[\frac{1}{4} F_{\mu \nu} F^{\mu \nu}-\frac{1}{2} D_{\mu} X_{I} D^{\mu} X^{I}+\frac{1}{4}\left[X_{I}, X_{J}\right]\left[X^{I}, X^{J}\right]\right. \\
+ & \left.\frac{1}{2} \overline{\lambda^{\mathbf{A}}} \gamma^{\mu} D_{\mu} \lambda^{\mathbf{A}}-\frac{1}{2} \overline{\lambda^{\mathbf{A}}}\left(\gamma^{I}\right)^{\mathbf{A}}{ }_{\mathbf{B}}\left[X_{I}, \lambda^{\mathbf{B}}\right]\right] .
\end{aligned}
$$

This Lagrangian has manifest $\mathrm{SO}(5) \simeq \mathrm{Sp}(2)$ symmetry. To connect the $\mathrm{Sp}(2)$-invariant Lagrangian (4.40) with (4.35), one has to decompose the $\mathrm{Sp}(2)$-Majorana fermion into two $\mathrm{Sp}(1)$-Majorana ones, which breaks manifest $\mathrm{Sp}(2)$ symmetry. Realizing the $\mathrm{SO}(5)$ gamma matrices $\gamma_{I}$ by a chiral expression, we can rewrite the $\operatorname{Sp}(2)$-Majorana condition given by $(4.39)$ as

$$
\lambda^{\mathbf{A}}=\left(\begin{array}{c}
\lambda_{+}^{A} \\
\lambda_{-}^{A}
\end{array}\right), \quad \lambda_{ \pm}^{A}= \pm \varepsilon^{A B} C_{5}{\overline{\lambda_{ \pm}^{B}}}^{T}
$$

where $A, B=1,2$. The Lagrangian (4.40) agrees with that given by (4.35) under an identification such that

$$
\lambda_{+}^{A}=\lambda_{1}^{A}, \quad \lambda_{-}^{A}=i \lambda_{2}^{A}, \quad X^{5}=\varphi .
$$


Five dimensional supersymmetry current can be obtained by dimensional reduction from ten dimensional supersymmetry current (2.5).

$$
\overline{S_{\rho}^{\mathbf{A}}}=\frac{1}{g_{5}^{2}} \operatorname{Tr}\left[\frac{1}{2} F_{\mu \nu} \overline{\lambda^{\mathbf{A}}} \gamma^{\rho} \gamma^{\mu \nu}-D_{\mu} X^{I} \overline{\lambda^{\mathbf{B}}}\left(\gamma^{I}\right)^{\mathbf{B}} \mathbf{A} \gamma^{\rho} \gamma^{\mu}-\frac{1}{2}\left[X^{I}, X^{J}\right] \overline{\lambda^{\mathbf{B}}} \gamma^{\rho}\left(\gamma^{I J}\right)^{\mathbf{B}} \mathbf{A}\right] .
$$

The $\mathcal{N}=2$ supersymmetry algebra in five dimensions is also computed by dimensional reduction of that of ten-dimensional supersymmetry algebra, which keeps manifest $\mathrm{SO}(5)$ symmetry. We neglect the contributions of fermions for simplicity. From (2.12) we calculate

$$
\begin{aligned}
\Delta \overline{S_{\rho}^{\mathbf{A}}}= & -2 T_{\rho \mu} \overline{\epsilon^{\mathbf{A}}} \gamma^{\mu}-2 i T_{\rho I} \overline{\epsilon^{\mathbf{B}}}\left(\gamma^{I}\right)^{\mathbf{B}}{ }_{\mathbf{A}}+\frac{1}{4 g_{5}^{2}} \operatorname{Tr}\left[F_{\lambda \sigma} F_{\mu \nu}\right] \varepsilon_{\rho}{ }^{\lambda \sigma \mu \nu} \overline{\epsilon^{\mathbf{A}}}-\frac{1}{g_{5}^{2}} \gamma_{\rho}^{\lambda \sigma \mu \nu} \partial_{\sigma} \operatorname{Tr}\left[X^{I} F_{\mu \nu}\right] \overline{\epsilon^{\mathbf{B}}} \gamma_{I}^{\mathbf{B}} \mathbf{A} \gamma_{\lambda} \\
& +\frac{1}{2 g_{5}^{2}} \gamma_{\rho}^{\lambda \sigma \mu \nu} \partial_{\mu} \operatorname{Tr}\left[X^{I} D_{\nu} X^{J}\right] \overline{\epsilon^{\mathbf{B}}} \gamma_{I J}{ }^{\mathbf{B}} \gamma_{\lambda \sigma}+\frac{1}{6 g_{5}^{2}} \partial_{\nu} \operatorname{Tr}\left[\left[X^{I}, X^{J}\right], X^{K}\right] \overline{\epsilon^{\mathbf{B}}} \gamma_{\rho}^{\nu} \varepsilon^{I J K L M} \gamma_{M L}{ }^{\mathbf{B}} \mathbf{A}
\end{aligned}
$$

where $\varepsilon_{12345}=1$,

$$
\begin{aligned}
& T_{\mu \rho}=\frac{1}{4 g_{5}^{2}} \operatorname{Tr}\left[g_{\mu \rho}\left(F_{\nu \sigma} F^{\nu \sigma}-2 D_{\nu} X_{I} D^{\nu} X^{I}+\left[X_{I}, X_{J}\right]\left[X^{I}, X^{J}\right]\right)+4\left(F_{\mu}{ }^{\nu} F_{\nu \rho}+D_{\mu} X^{I} D_{\rho} X_{I}\right)\right], \\
& T_{\rho I}=\frac{i}{g_{5}^{2}} \partial_{\nu} \operatorname{Tr}\left[F_{\rho}{ }^{\nu} X_{I}\right] .
\end{aligned}
$$

The supercharge with $\mathrm{Sp}(2)$ index is

$$
Q^{\mathbf{A}}=\int d^{4} x S^{0 \mathbf{A}}
$$

and $\Delta \mathcal{O}=-i\left[\epsilon^{\mathbf{B}} Q^{\mathbf{B}}, \mathcal{O}\right]$. Then the local supersymmetry algebra of $\mathcal{N}=2 \mathrm{SYM}$ is

$$
\begin{aligned}
\left\{Q^{\mathbf{B}}, \overline{S_{\rho}^{\mathbf{A}}}\right\}= & -2 i T_{\rho \mu} \delta_{\mathbf{A}}^{\mathbf{B}} \gamma^{\mu}+2 T_{\rho I}\left(\gamma^{I}\right)^{\mathbf{B}} \mathbf{A}+j_{\rho} \delta_{\mathbf{A}}^{\mathbf{B}}+j_{\rho \lambda}^{I} \gamma_{I}^{\mathbf{B}} \mathbf{A} \gamma^{\lambda} \\
& +j_{\rho \lambda \sigma}^{I J} \gamma_{I J} \mathbf{B}_{\mathbf{A}} \gamma^{\lambda \sigma}+j_{\rho \mu \sigma \lambda}^{L M} \gamma^{\lambda \sigma \mu} \gamma_{M L} \mathbf{B}_{\mathbf{A}}
\end{aligned}
$$

where

$$
\begin{aligned}
j_{\rho \lambda}^{I} & =-\frac{i}{g_{5}^{2}} \gamma_{\rho \lambda}{ }^{\sigma \mu \nu} \partial_{\sigma} \operatorname{Tr}\left[X^{I} F_{\mu \nu}\right], \\
j_{\rho \lambda \sigma}^{I J} & =\frac{i}{2 g_{5}^{2}} \gamma_{\rho \lambda \sigma}{ }^{\mu \nu} \partial_{\mu} \operatorname{Tr}\left[X^{I} D_{\nu} X^{J}\right], \\
j_{\rho \mu \sigma \lambda}^{L M} & =\frac{i}{36 g_{5}^{2}} \partial^{\nu} \operatorname{Tr}\left[\left[X_{I}, X_{J}\right], X_{K}\right] \gamma_{\rho \nu \mu \sigma \lambda} \varepsilon^{I J K L M} .
\end{aligned}
$$

Thus supersymmetry algebra is computed as

$$
\begin{aligned}
\left\{Q^{\mathbf{B}}, \overline{Q^{\mathbf{A}}}\right\}= & -2 i P_{\mu} \gamma^{\mu} \delta_{\mathbf{A}}^{\mathbf{B}}+2 P_{I}\left(\gamma^{I}\right)^{\mathbf{B}} \mathbf{A}+Z \delta_{\mathbf{A}}^{\mathbf{B}}+Z_{\lambda}^{I} \gamma_{I}^{\mathbf{B}} \mathbf{A} \gamma^{\lambda} \\
& +Z_{\lambda \sigma}^{I J} \gamma_{I J} \mathbf{B}_{\mathbf{A}} \gamma^{\lambda \sigma}+Z_{\mu \sigma \lambda}^{L M} \gamma^{\lambda \sigma \mu} \gamma_{M L} \mathbf{B}_{\mathbf{A}}
\end{aligned}
$$

where we set

$$
P^{\mu}=\int d^{4} x T^{0 \mu}, \quad P^{I}=\int d^{4} x T^{0 I},
$$




$$
Z_{\lambda}^{I}=\int d^{4} x j_{\lambda}^{I 0}, \quad Z_{\lambda \sigma}^{I J}=\int d^{4} x j_{\lambda \sigma}^{I J 0}, \quad Z_{\mu \sigma \lambda}^{L M}=\int d^{4} x j^{L M \sigma}{ }_{\mu \sigma \lambda} .
$$

This result agrees with that in [18] up to the conventions, except the quadratic and quartic terms of the scalar fields. In [18] the quartic term remains as $Z_{0}^{i}$, though it always vanishes in our results, which is required for conservation of supercurrent or supercharge. Another one is the relative coefficients of the term $X^{I} D_{\nu} X^{J}$ mismatch between those results. Note that these mismatches do not affect the analysis done in [18].

\section{Superalgebra in 4d SYM}

In this section we investigate superalgebras in four dimensional SYM by performing dimensional reduction for higher dimensional SYM studied in the previous sections. The torus compactification gives four dimensional $\mathcal{N}=2 \mathrm{SYM}$ with the kinetic term canonical. Thus strictly speaking our study is not so general as to apply to general $\mathcal{N}=2 \mathrm{SYM}$, which admit exact analysis to turn out to have rich structure of SUSY QFT $[29,33]$ and are to be obtained by Riemann surface compactification of six dimensional $(2,0)$ SCFT describing an M-five brane [34, 35]. However relying on the Lagrangian description it becomes possible to give an explicit expression for a generic form of $\mathcal{N}=2$ superalgebra including fermionic contributions. Especially on an instanton or monopole background there exist fermionic zero modes [36-40], which can a priori affect the central charge formula in Coulomb branch [41]. Our analysis implies that contribution of such a fermionic zero mode vanishes in the superalgebra. In addition our study provides another method to derive extended supersymmetry algebra in four dimensions, which may be simpler than direct computation in canonical formalism especially for the fermionic part [31].

\section{$5.1 \mathcal{N}=2$ vector multiplet}

First we consider $\mathcal{N}=2 \mathrm{SYM}$ consisting of an $\mathcal{N}=2$ vector multiplet, which can be obtained by six dimensional SYM studied in section 3.1. We compactify six dimensional theory in $x^{4}, x^{5}$ directions. Degeneration of the two tori leads to two real scalar fields from the gauge fields of these direction. We combine them to be a complex scalar field so that

$$
\phi=\frac{A_{4}-i A_{5}}{\sqrt{2}} .
$$

We decompose six dimensional gamma matrices into four dimensional ones by

$$
\Gamma_{\mu}=\gamma_{\mu} \otimes \mathbf{1}, \quad \Gamma_{4}=\hat{\gamma} \otimes \sigma_{1}, \quad \Gamma_{5}=\hat{\gamma} \otimes \sigma_{2}
$$

where $\hat{\gamma}=i \gamma_{0123}$ is a chirality matrix in four dimensions. Then six dimensional chirality matrix and charge conjugation matrix can be computed as

$$
\hat{\Gamma}=\hat{\gamma} \otimes \sigma_{3}, \quad C_{6}=C_{4} \hat{\gamma} \otimes i \sigma_{2}
$$

where $C_{4}=-i \gamma_{03}$. Thus six dimensional symplectic-Majorana Weyl fermion constrained by (3.1), which we denote by $\lambda_{6 d}$, is decomposed as follows.

$$
\lambda_{6 d}^{A}=\left(\begin{array}{c}
\lambda_{+}^{A} \\
\lambda_{-}^{A}
\end{array}\right), \quad \hat{\gamma} \lambda_{ \pm}^{A}= \pm \lambda_{ \pm}^{A}, \quad \lambda_{ \pm}^{A}=\varepsilon^{A B} C_{4}\left(\overline{\lambda_{\mp}^{B}}\right)^{T} .
$$


By using the last condition above, we can always write the fermionic part of the theory only in terms of $\lambda_{+}^{A}$. From (3.3) we obtain the Lagrangian of $\mathcal{N}=2$ SYM as

$$
\begin{aligned}
\mathcal{L}_{V}= & \frac{1}{g_{4}^{2}} \operatorname{Tr}\left[\overline{\lambda_{+}^{A}}\left[\not D, \lambda_{+}^{A}\right]+\frac{1}{4} F_{\mu \nu} F^{\mu \nu}-D_{\mu} \phi^{\dagger} D^{\mu} \phi+\frac{1}{2} D_{B}^{A} D^{B}{ }_{A}-\frac{1}{2}\left[\phi, \phi^{\dagger}\right]^{2}\right. \\
& \left.+\frac{1}{\sqrt{2}}\left(-\varepsilon^{A B} \overline{\lambda_{+}^{A}} C_{4}\left[\phi,{\overline{\lambda_{+}^{B}}}^{T}\right]+\varepsilon_{A B}\left(\lambda_{+}^{B}\right)^{T} C_{4}\left[\phi^{\dagger}, \lambda_{+}^{A}\right]\right)\right]
\end{aligned}
$$

where $g_{4}$ is a coupling constant of this theory. The $\mathcal{N}=2$ supersymmetry transformation rule is computed from (3.4). The supersymmetry parameter denoted by $\epsilon_{6 d}^{A}$ is subject to similar decomposition to gaugino in (5.4) so that

$$
\epsilon_{6 d}^{A}=\left(\begin{array}{c}
\epsilon_{+}^{A} \\
\epsilon_{-}^{A}
\end{array}\right), \quad \hat{\gamma} \epsilon_{ \pm}^{A}= \pm \epsilon_{ \pm}^{A}, \quad \epsilon_{ \pm}^{A}=-\varepsilon^{A B} C_{4}\left(\overline{\epsilon_{\mp}^{B}}\right)^{T} .
$$

Eliminating $\epsilon_{-}^{A}$ by using the last equation of (5.6) we obtain $\mathcal{N}=2$ SUSY transformation.

$$
\begin{aligned}
\Delta A_{\mu} & =\overline{\epsilon_{+}^{A}} \gamma_{\mu} \lambda_{+}^{A}+\overline{\lambda_{+}^{A}} \gamma_{\mu} \epsilon_{+}^{A}, \\
\Delta \phi & =\sqrt{2} \varepsilon_{A B}\left(\epsilon_{+}^{B}\right)^{T} C_{4} \lambda_{+}^{A}, \\
\Delta \lambda_{+}^{A}= & \frac{1}{2} F_{\mu \nu} \gamma^{\mu \nu} \epsilon_{+}^{A}+\sqrt{2}[\not D, \phi] \varepsilon^{A B} C_{4}{\overline{\epsilon_{+}^{B}}}^{T}-\left[\phi, \phi^{\dagger}\right] \epsilon_{+}^{A}+\alpha D^{A} \epsilon_{+}^{B}, \\
\Delta D_{B}^{A}= & \alpha\left(D_{\mu} \overline{\lambda_{+}^{B}} \gamma^{\mu} \epsilon_{+}^{A}-\overline{\epsilon_{+}^{B}} \gamma^{\mu} D_{\mu} \lambda_{+}^{A}-\sqrt{2} \varepsilon_{B C}\left[\phi^{\dagger},\left(\lambda_{+}^{C}\right)^{T}\right] C_{4} \epsilon_{+}^{A}+\sqrt{2} \varepsilon^{A C}\left[\phi, \overline{\lambda_{+}^{B}}\right] C_{4}{\overline{\epsilon_{+}^{C}}}^{T}\right. \\
& \left.-\frac{1}{2} \delta_{B}^{A}\left(D_{\mu} \overline{\lambda_{+}^{C}} \gamma^{\mu} \epsilon_{+}^{C}-\overline{\epsilon_{+}^{C}} \gamma^{\mu} D_{\mu} \lambda_{+}^{C}-\sqrt{2} \varepsilon_{D C}\left[\phi^{\dagger},\left(\lambda_{+}^{C}\right)^{T}\right] C_{4} \epsilon_{+}^{D}+\sqrt{2} \varepsilon^{D C}\left[\phi, \overline{\lambda_{+}^{D}}\right] C_{4}{\overline{\epsilon_{+}^{C}}}^{T}\right)\right),
\end{aligned}
$$

where $\alpha$ is arbitrary when one considers only a vector multiplet though $\alpha=\sqrt{2}$ when we also consider coupling of a hyper multiplet, which is introduced in the next subsection. The supersymmetry current in this theory is computed from (3.5). The result is

$$
\begin{aligned}
& \overline{S_{\rho}^{A}}=\operatorname{Tr}\left[\frac{1}{2} F_{\mu \nu} \overline{\lambda_{+}^{A}} \gamma^{\rho} \gamma^{\mu \nu}+\sqrt{2} \varepsilon_{A B}\left(\lambda_{+}^{B}\right)^{T} C_{4} D_{\mu} \phi^{\dagger} \gamma^{\rho} \gamma^{\mu}-\overline{\lambda_{+}^{A}} \gamma^{\rho}\left[\phi, \phi^{\dagger}\right]\right], \\
& S_{\rho}^{A}=\operatorname{Tr}\left[\frac{1}{2} F_{\mu \nu} \gamma^{\mu \nu} \gamma^{\rho} \lambda_{+}^{A}-\sqrt{2} D_{\mu} \phi \gamma^{\mu} \gamma^{\rho} \varepsilon^{A B} C_{4}\left(\overline{\lambda_{+}^{B}}\right)^{T}+\left[\phi, \phi^{\dagger}\right] \gamma^{\rho} \lambda_{+}^{B}\right] .
\end{aligned}
$$

From the supercurrent we obtain supercharge in four dimensions

$$
Q^{A}=\int d^{3} x S^{0 A}
$$

then $\Delta \mathcal{O}=-i\left[\overline{\epsilon_{+}^{B}} Q^{B}+\overline{Q^{B}} \epsilon_{+}^{B}, \mathcal{O}\right]$. Performing dimensional reduction for (3.36) we obtain local form of supersymmetry algebra of four dimensional SYM.

$$
\begin{aligned}
\left\{Q^{B}, \overline{S_{\rho}^{A}}\right\} & =\left(\left(-2 i T_{\rho \mu}+j_{\rho \mu}+j_{\rho \mu}^{\prime}\right) \delta_{A}^{B}+j_{A \rho \mu}^{B}\right) \gamma^{\mu}, \\
\left\{{\overline{Q^{B}}}^{T}, \overline{S_{\rho}^{A}}\right\} & =\varepsilon_{A B}\left(2 i T_{\rho}-j_{\rho}-j_{\rho}^{\prime}\right) C_{4}, \\
\left\{{\overline{Q^{B}}}^{T},\left(S_{\rho}^{A}\right)^{T}\right\} & =\left(\left(-2 i T_{\rho \mu}-j_{\rho \mu}+j_{\rho \mu}^{\prime}\right) \delta_{B}^{A}-j_{B \rho \mu}^{A}\right)\left(\gamma^{\mu}\right)^{T},
\end{aligned}
$$




$$
\left\{Q^{B},\left(S_{\rho}^{A}\right)^{T}\right\}=\varepsilon^{B A}\left(2 i T_{\rho}-j_{\rho}-j_{\rho}^{\prime}\right)^{\dagger} C_{4},
$$

where

$$
\begin{aligned}
T_{\mu \rho}= & \frac{1}{4 g_{4}^{2}} \operatorname{Tr}\left[g_{\mu \rho}\left(F_{\nu \sigma} F^{\nu \sigma}-4 D_{\nu} \phi D^{\nu} \phi^{\dagger}-2\left[\phi, \phi^{\dagger}\right]^{2}\right)+4\left(F_{\mu}^{\nu} F_{\nu \rho}+2 D_{(\mu} \phi D_{\rho)} \phi\right)\right. \\
& \left.-2\left(\overline{\lambda_{+}^{A}} \gamma_{\rho} D_{\mu} \lambda_{+}^{A}-D_{\mu} \overline{\lambda_{+}^{A}} \gamma_{\rho} \lambda_{+}^{A}\right)\right] \\
T_{\rho}= & -\frac{\sqrt{2}}{g_{4}^{2}} \partial_{\nu} \operatorname{Tr}\left[F_{\rho}^{\nu} \phi^{\dagger}\right]+\frac{1}{2 g_{4}^{2}} \varepsilon^{A B} \operatorname{Tr}\left[\overline{\lambda_{+}^{A}} C_{4} D_{\rho}\left(\overline{\lambda_{+}^{B}}\right)^{T}\right] \\
& +\frac{\sqrt{2}}{4 g_{4}^{2}}\left(\operatorname{Tr}\left[\overline{\lambda_{+}^{A}} \gamma_{\rho}\left[\phi^{\dagger}, \lambda_{+}^{A}\right]-\left[\phi^{\dagger}, \overline{\lambda_{+}^{A}}\right] \gamma_{\rho} \lambda_{+}^{A}\right]\right) \\
j_{\rho \mu}= & -\frac{1}{g_{4}^{2}} \partial_{\lambda} \operatorname{Tr}\left[\phi D_{\nu} \phi-\phi^{\dagger} D_{\nu} \phi^{\dagger}\right] i \sigma_{\rho \mu}^{\lambda \nu} \\
j_{\rho}= & -\frac{\sqrt{2}}{g_{4}^{2}} \partial_{\lambda} \operatorname{Tr}\left[\phi^{\dagger} F_{\mu \nu}\right] i \sigma_{\rho}^{\lambda \mu \nu} \\
j_{\rho}^{\prime}= & -\frac{i}{g_{4}^{2}} \varepsilon^{C D} \partial^{\mu} \operatorname{Tr}\left[\overline{\lambda_{+}^{C}} \gamma_{\rho \mu} C_{4} \overline{\lambda_{+}^{D^{2}}}\right] \\
j_{A}^{B}{ }_{\rho \mu}= & \frac{2 i}{g_{4}^{2}} \partial_{\mu} \operatorname{Tr}\left[\overline{\lambda_{+}^{A}} \gamma_{\rho} \lambda_{+}^{B}-\frac{1}{2} \delta_{A}^{B} \overline{\lambda_{+}^{C}} \gamma_{\rho} \lambda_{+}^{C}\right] \\
j_{\rho \nu}^{\prime}= & -\frac{i}{g_{4}^{2}} \sigma_{\rho \nu \mu \sigma} \partial^{\mu} \operatorname{Tr}\left[\overline{\lambda_{+}^{C}} \gamma^{\sigma} \lambda_{+}^{C}\right]
\end{aligned}
$$

with $\sigma_{0123}=-i$. Note that in the bosonic terms there exists a brane current which describes one dimensional object (string) as $j_{\rho \mu}{ }^{6}$ Performing volume integration for both sides, we obtain supersymmetry algebra in four dimensional $\mathcal{N}=2 \mathrm{SYM}$.

$$
\begin{aligned}
\left\{Q^{B}, \overline{Q^{A}}\right\} & =\left(\left(-2 i P_{\mu}+Z_{\mu}+Z_{\mu}^{\prime}\right) \delta_{A}^{B}+Z_{A \mu}^{B}\right) \gamma^{\mu}, \\
\left\{\overline{Q^{B}}, \overline{Q^{A}}\right\} & =\varepsilon_{A B}\left(2 i \mathcal{P}-Z-Z^{\prime}\right) C_{4}, \\
\left\{{\overline{Q^{B}}}^{T},\left(Q^{A}\right)^{T}\right\} & =\left(\left(-2 i P_{\mu}-Z_{\mu}+Z_{\mu}^{\prime}\right) \delta_{B}^{A}-Z_{B \mu}^{A}\right)\left(\gamma^{\mu}\right)^{T}, \\
\left\{Q^{B},\left(Q^{A}\right)^{T}\right\} & =\varepsilon^{B A}\left(-2 i \mathcal{P}^{\dagger}-Z^{\dagger}-Z^{\prime \dagger}\right) C_{4},
\end{aligned}
$$

where we set

$$
\begin{aligned}
& P^{\mu}=\int d^{3} x T^{0 \mu}, \quad \mathcal{P}=\int d^{3} x T^{0}, \quad Z=\int d^{3} x j^{0}, \quad Z_{\lambda}=\int d^{3} x j^{0}{ }_{\lambda}, \\
& Z_{A \mu}^{\prime B}=\int d^{3} x j_{A}^{\prime B 0}{ }_{\mu}, \quad Z_{\mu}^{\prime}=\int d^{3} x j^{\prime 0}{ }_{\mu}, \quad Z^{\prime}=\int d^{3} x j^{\prime 0} .
\end{aligned}
$$

This result shows that contributions of fermion zero modes to the superalgebra vanishes. This is because a fermionic zero mode (on an instanton background) is essentially given by

\footnotetext{
${ }^{6}$ Although improvement transformations keeping $\mathcal{N}=2$ SUSY are not known, those in $\mathcal{N}=1$ were studied in [32], which suggests that Schwinger terms in a superalgebra can be reabsorbed if it behaves suitably at the boundary. Thus since $j_{\rho \mu}, j_{\rho}, j_{\rho \mu}^{\prime}$ in our superalgebra are Schwinger terms, these may be reabsorbed into an improvement transformation. However, these are not always removable because, for example, $j_{\rho}$ measures a background magnetic charge in the Coulomb phase and affects the physical central charge. We leave a problem to clarify whether other Schwinger terms are physical to future works.
} 
a shift generated by SUSY transformation [40], $\lambda \sim F_{\mu \nu} \gamma^{\mu \nu} \epsilon_{0}$ with $\epsilon_{0}$ a constant spinor, which implies that the zero mode scales as $r^{-2}$ near the boundary $r \sim \infty$.

In particular we obtain the famous formula of central charge as

$$
\left\{{\overline{Q^{B}}}^{T}, \overline{Q^{A}}\right\}=2 \sqrt{2} \varepsilon_{A B} \mathcal{Z} C_{4}
$$

where

$$
\mathcal{Z}=\frac{1}{\sqrt{2}}\left(i \mathcal{P}-\frac{1}{2} Z-\frac{1}{2} Z^{\prime}\right)
$$

For example, let us consider SYM with SU(2) gauge group in the Coulomb branch.

$$
\phi=a^{*} \sigma_{3}, \quad \lambda^{A}=0,
$$

where $a$ is a complex number. In electrically and magnetically charged background such that

$$
n_{e}=\frac{1}{g_{4}^{2}} \int d^{3} x \partial_{i}\left(i f^{i 0}\right), \quad n_{m}=\frac{1}{4 \pi} \int d^{3} x \partial_{i}\left(i \frac{1}{2} \varepsilon^{i j k} f_{j k}\right),
$$

where $f_{\mu \nu}=\operatorname{Tr}\left[F_{\mu \nu} \sigma_{3}\right]$, one can show that the central charge is computed as

$$
\mathcal{Z}=n_{e} a+n_{m} a_{D}
$$

where $a_{D}=\tau_{0} a$ with the holomorphic coupling $\tau_{0}=\frac{4 \pi i}{g_{4}^{2}} .7$ This gives the same formula as in [33].

\subsection{Inclusion of a hyper multiplet}

In this subsection we study four dimensional $\mathcal{N}=2 \mathrm{SYM}$ including a hyper multiplet by dimensional reduction. We use the same notation for two complex scalar fields $q^{A}$. A six dimensional chiral fermion $\psi_{6 d}$ reduces to

$$
\psi_{6 d}=\left(\begin{array}{c}
\psi_{-} \\
\psi_{+}
\end{array}\right), \quad \hat{\gamma} \psi_{ \pm}= \pm \psi_{ \pm},
$$

Then the Lagrangian of a hyper multiplet reduces to

$$
\begin{aligned}
\mathcal{L}_{H}= & -D_{\mu}\left(q^{A}\right)^{\dagger} D^{\mu} q^{A}+\frac{1}{2}\left(\overline{\psi_{+}} \not D \psi_{+}+\overline{\psi_{-}} \not D \psi_{-}\right)+\sqrt{2}\left(q^{A}\right)^{\dagger} D^{A}{ }_{B} q^{B} \\
& +\varepsilon^{A B}\left(q^{A}\right)^{\dagger} \overline{\lambda_{+}^{B}} \psi_{-}+\left(q^{A}\right)^{\dagger}\left(\lambda_{+}^{A}\right)^{T} C_{4} \psi_{+}+\varepsilon_{A B} \overline{\psi_{-}} \lambda_{+}^{B} q^{A}-\overline{\psi_{+}} C_{4}\left(\overline{\lambda_{+}^{A}}\right)^{T} q^{A} \\
& +\frac{1}{\sqrt{2}}\left(\overline{\psi_{-}} \phi \psi_{+}+\overline{\psi_{+}} \phi^{\dagger} \psi_{-}\right)-\left(q^{A}\right)^{\dagger}\left\{\phi, \phi^{\dagger}\right\} q^{A} .
\end{aligned}
$$

The SUSY transformation boils down to

$$
\begin{aligned}
\Delta q^{A} & =\varepsilon^{A B} \overline{\epsilon_{+}^{B}} \psi_{-}-\left(\epsilon_{+}^{A}\right)^{T} C_{4} \psi_{+}, \\
\Delta \psi_{-} & =2\left(\gamma^{\mu} \varepsilon_{B A} \epsilon_{+}^{B} D_{\mu} q^{A}+\sqrt{2} C_{4} \overline{\epsilon_{+}^{A}} \phi q^{A}\right),
\end{aligned}
$$

\footnotetext{
${ }^{7}$ The real part of the holomorphic coupling appears once the topological term $F \wedge F$ is introduced.
} 


$$
\Delta \psi_{+}=2\left(-\gamma^{\mu} C_{4}{\overline{\epsilon_{+}^{A}}}^{T} D_{\mu} q^{A}-\sqrt{2} \varepsilon_{B A} \epsilon_{+}^{B} \phi^{\dagger} q^{A}\right) .
$$

The supercurrent is computed from six dimensional one (3.28). Since the part of a vector multiplet was already computed as (5.8), we have only to compute the part of a hyper multiplet. The result is

$$
\begin{aligned}
\overline{S_{\rho}^{A} \text { hyp }}= & \varepsilon_{A B} \overline{\psi_{-}} \gamma_{\rho} \gamma^{\mu} D_{\mu} q^{B}+\varepsilon_{A B} \overline{\psi_{+}} \gamma_{\rho}\left(-\sqrt{2} \phi^{\dagger}\right) q^{B}+\left(D_{\mu} q^{A}\right)^{\dagger} \psi_{+}^{T} C_{4} \gamma_{\rho} \gamma^{\mu}+\left(q^{A}\right)^{\dagger} \sqrt{2} \phi^{\dagger} \psi_{-}^{T} C_{4} \gamma_{\rho} \\
& -2\left(q^{A}\right)^{\dagger} \overline{\lambda_{+}^{B}} \gamma_{\rho} q^{B}+\left(q^{B}\right)^{\dagger} \overline{\lambda_{+}^{A}} \gamma_{\rho} q^{B}, \\
S_{\rho \text { hyp }}^{A}= & -\gamma^{\mu} \gamma_{\rho} C_{4}{\overline{\psi_{+}}}^{T} D_{\mu} q^{A}+\gamma_{\rho} C_{4} \bar{\psi}_{-}^{T} \sqrt{2} \phi q^{A}+\left(D_{\mu} q^{A}\right)^{\dagger} \gamma^{\mu} \gamma_{\rho} \psi_{-}+\left(q^{A}\right)^{\dagger} \sqrt{2} \phi \gamma_{\rho} \psi_{+} \\
& -2\left(q^{B}\right)^{\dagger} \gamma_{\rho} \lambda_{+}^{A} q^{B}+\left(q^{B}\right)^{\dagger} \gamma_{\rho} \lambda_{+}^{A} q^{B} .
\end{aligned}
$$

Supercharge in four dimensions is given by (5.9). From (3.36) we obtain local supersymmetry algebra of four dimensional SYM including the contribution of a hyper multiplet.

$$
\begin{aligned}
\left\{Q^{B}, \overline{S_{\rho}^{A}}\right\} & =\left(\left(-2 i T_{\rho \mu}+j_{\rho \mu}\right) \delta_{A}^{B}+\left(j_{A \rho \mu}^{B}+c_{A \rho \mu}^{B}\right)\right) \gamma^{\mu}, \\
\left\{\overline{Q^{B}}, \overline{S_{\rho}^{A}}\right\} & =\varepsilon_{A B}\left(2 i T_{\rho}-j_{\rho}\right) C_{4}-\varepsilon_{C B} c_{A \rho \sigma \lambda}^{C} C_{4} \gamma^{\lambda \sigma}, \\
\left\{{\overline{Q^{B}}}^{T},\left(S_{\rho}^{A}\right)^{T}\right\} & =\left(\left(-2 i T_{\rho \mu}-j_{\rho \mu}\right) \delta_{B}^{A}-\left(j_{B \rho \mu}^{A}+c_{A \rho \mu}^{B}\right)\right)\left(\gamma^{\mu}\right)^{T}, \\
\left\{Q^{B},\left(S_{\rho}^{A}\right)^{T}\right\} & =\varepsilon^{B A}\left(2 i T_{\rho}-j_{\rho}\right)^{\dagger} C_{4}-\varepsilon^{C A}\left(c_{B \rho \sigma \lambda}^{C}\right)^{\dagger} \gamma^{\lambda \sigma} C_{4},
\end{aligned}
$$

where

$$
\begin{aligned}
T_{\mu \rho}= & \frac{1}{4 g_{4}^{2}} \operatorname{Tr}\left[g_{\mu \rho}\left(F_{\nu \sigma} F^{\nu \sigma}-4 D_{\nu} \phi D^{\nu} \phi^{\dagger}-2\left[\phi, \phi^{\dagger}\right]^{2}\right)+4\left(F_{\mu}{ }^{\nu} F_{\nu \rho}+2 D_{(\mu} \phi D_{\rho)} \phi\right)\right] \\
& +2 D_{(\mu}\left(q^{A}\right)^{\dagger} D_{\rho)} q^{A}-g_{\mu \rho} \partial_{\nu}\left(\left(q^{A}\right)^{\dagger} D^{\nu} q^{A}\right) \\
T_{\rho}= & \frac{-\sqrt{2}}{g_{4}^{2}} \partial_{\nu} \operatorname{Tr}\left[F_{\rho}^{\nu} \phi^{\dagger}\right]-\sqrt{2} D_{\rho}\left(q^{A}\right)^{\dagger} \phi^{\dagger} q^{A}+\sqrt{2}\left(q^{A}\right)^{\dagger} \phi^{\dagger} D_{\rho} q^{A} \\
c_{A}^{B \rho \lambda}= & 4 i \partial_{\mu}\left[\left(q^{A}\right)^{\dagger} D_{\nu} q^{B}-\frac{1}{2} \delta_{A}^{B}\left(q^{C}\right)^{\dagger} D_{\nu} q^{C}\right] \sigma^{\rho \mu \nu \lambda} \\
c_{A}^{B \rho \sigma \lambda}= & -2 i \sqrt{2} \partial_{\mu}\left[\left(q^{A}\right)^{\dagger} \phi^{\dagger} q^{B}-\frac{1}{2} \delta_{A}^{B}\left(q^{C}\right)^{\dagger} \phi^{\dagger} q^{C}\right] \sigma^{\rho \mu \sigma \lambda}
\end{aligned}
$$

This leads to supersymmetry algebra in four dimensional $\mathcal{N}=2 \mathrm{SYM}$.

$$
\begin{aligned}
\left\{Q^{B}, \overline{Q^{A}}\right\} & =\left(\left(-2 i P_{\mu}+Z_{\mu}\right) \delta_{A}^{B}+\left(Z_{A \mu}^{B}+y_{A \mu}^{B}\right)\right) \gamma^{\mu}, \\
\left\{\overline{Q^{B}}{ }^{T}, \overline{Q^{A}}\right\} & =\varepsilon_{A B}(2 i \mathcal{P}-Z) C_{4}-\varepsilon_{C B} y_{A}^{C \mu \nu} C_{4} \gamma_{\nu \mu}, \\
\left\{{\overline{Q^{B}}}^{T},\left(Q^{A}\right)^{T}\right\} & =\left(\left(-2 i P_{\mu}-Z_{\mu}\right) \delta_{B}^{A}-\left(Z_{B \mu}^{A}+y_{A \mu}^{B}\right)\right)\left(\gamma^{\mu}\right)^{T}, \\
\left\{Q^{B},\left(Q^{A}\right)^{T}\right\} & =\varepsilon^{B A}\left(-2 i \mathcal{P}^{\dagger}-Z^{\dagger}\right) C_{4}-\varepsilon^{C A}\left(y_{B}^{C \mu \nu}\right)^{\dagger} \gamma_{\nu \mu} C_{4},
\end{aligned}
$$

where we set

$$
y_{A \mu}^{B}=\int d^{3} x c_{A}^{B 0}{ }_{\mu}, \quad y_{A \mu \nu}^{B}=\int d^{3} x c_{A \mu \nu}^{B 0} .
$$

Due to inclusion of a hyper multiplet there appear brane currents $c_{A}^{B \rho \lambda}, c_{A}^{B \rho \sigma \lambda}$ in the local form of superalgebra and corresponding brane charges $y_{A}^{B \lambda}, y_{A \mu \nu}^{B}$ in the superalgebra. 
One can include a complex mass $m$ of a hyper multiplet in this superalgebra by shifting the adjoint scalar field in the vector multiplet in a way that $\phi \rightarrow \frac{m}{\sqrt{2}}+\phi$. Under this shift $T_{\rho} \rightarrow T_{\rho}-i m J_{\rho}$, where $J_{\rho}=i\left(q^{A}\right)^{\dagger} D_{\rho} q^{A}-i D_{\rho}\left(q^{A}\right)^{\dagger} q^{A}$ is the U(1) flavor current. Thus the formula of central charge (5.28) is changed as

$$
\mathcal{Z}=\frac{1}{\sqrt{2}}\left(i \mathcal{P}+m F-\frac{1}{2} Z\right)
$$

where $F=\int d^{3} x J^{0}$ is the $\mathrm{U}(1)$ flavor charge. ${ }^{8}$

Let us compute this central charge with $\mathrm{SU}(2)$ gauge group in the Coulomb branch. The Kaluza-Klein momentum is computed as

$$
\mathcal{P}=-i \sqrt{2} a N_{e}
$$

where we used the equation of motion of the gauge field and set

$$
N_{e}=\frac{2}{g_{4}^{2}} \int d^{3} x \partial_{i}\left(i f^{i 0}\right)
$$

Remark that the hyper multiplet contributes to the Kaluza-Klein momentum so that the electric charge is twice as great as that in pure $\mathcal{N}=2$ SYM case with the form of central charge fixed. ${ }^{9}$ The Dirac quantization condition requires us to redefine the magnetic charge to be half compared to the pure SYM case.

$$
N_{m}=\frac{1}{8 \pi} \int d^{3} x \partial_{i}\left(i \frac{1}{2} \varepsilon^{i j k} f_{j k}\right)
$$

Then $Z$ is computed as

$$
Z=-2 \sqrt{2} a_{D} N_{m}
$$

where $a_{D}=\tau a$ with $\tau=\frac{8 \pi i}{g_{4}^{2}}$. Finally the central charge is obtained as

$$
\mathcal{Z}=N_{e} a+N_{m} a_{D}+\frac{1}{\sqrt{2}} F m
$$

which matches the formula given in [29] with the same normalization of the holomorphic coupling $\tau$. This normalization of the holomorphic coupling is important for the $\mathcal{N}=2$ SU(2) SYM with four flavors to enjoy SL(2, Z) symmetry [29] as well as to obtain the correct moduli space of $\mathcal{N}=2 \mathrm{SU}(3) \mathrm{SYM}$ with six flavors as the enlarged fundamental region in the upper half complex plain, in which the genuine strong coupling limit exists as $\operatorname{Im} \tau \rightarrow 0$ [42].

\footnotetext{
${ }^{8}$ In multiple flavor case, this changes as $\mathcal{Z}=\frac{1}{\sqrt{2}}\left(i \mathcal{P}+m_{i} F^{i}-\frac{1}{2} Z\right)$, where $m_{i}$ is the mass of $i$ th hyper multiplet and $F_{i}$ is the flavor $\mathrm{U}(1)$ charge of the $i$ th hyper multiplet.

${ }^{9}$ The factor two in (5.54) does not depend on the number of hyper multiplets.
} 


\section{$5.3 \mathcal{N}=4$ superalgebra}

In this final subsection we determine superalgebra in $\mathcal{N}=4 \mathrm{SYM}$ by dimensional reduction for ten dimensional SYM. By compactifying six directions $x^{3+m}$, where $m=1,2, \cdots, 6$, the gauge fields of these directions become scalar fields, which we denote by $X_{m}=-i A_{3+m}$. Accordingly we decompose the ten-dimensional gamma matrices as

$$
\Gamma_{\mu}=\gamma_{\mu} \otimes 1, \quad \Gamma_{m}=\hat{\gamma} \otimes \gamma_{m}
$$

where $\gamma_{m}$ are $\mathrm{SO}(6)$ gamma matrices, respectively. Since $\mathcal{N}=4 \mathrm{SYM}$ has $\mathrm{SU}(4) \mathrm{R}-$ symmetry, it is convenient to rewrite the $\mathrm{SO}(6)$ vector representation by $\mathrm{SU}(4)$ antisymmetric representation.

$$
\gamma_{a 4}=\frac{1}{2}\left(\gamma_{a}-i \gamma_{a+3}\right), \quad \gamma_{a b}=\varepsilon_{a b c}\left(\gamma_{c 4}\right)^{\dagger}
$$

where $a, b, c=1,2,3$. These satisfy

$$
\gamma_{\mathbf{A B}}=-\gamma_{\mathbf{B A}}, \quad \gamma^{\mathbf{A B}}=\frac{1}{2} \varepsilon^{\mathbf{A B C D}} \gamma_{\mathbf{C D}}=\left(\gamma_{\mathbf{A B}}\right)^{\dagger}
$$

where $\mathbf{A}, \mathbf{B}, \mathbf{C}, \mathbf{D}=1,2,3,4$. We do the same thing for $X^{m} \cdot \gamma_{\mathbf{A B}}$ is explicitly realized as

$$
\gamma_{\mathbf{A B}}=\left(\begin{array}{cc}
0 & -\tilde{\rho}_{\mathbf{A B}} \\
\rho_{\mathbf{A B}} & 0
\end{array}\right)
$$

where

$$
\left(\rho_{\mathbf{A B}}\right)^{\mathbf{C D}}=\delta_{\mathbf{A}}^{\mathbf{C}} \delta_{\mathbf{B}}^{\mathbf{D}}-\delta_{\mathbf{B}}^{\mathbf{C}} \delta_{\mathbf{A}}^{\mathbf{D}}, \quad\left(\tilde{\rho}_{\mathbf{A B}}\right)_{\mathbf{C D}}=\varepsilon_{\mathbf{A B C D}} .
$$

Then the ten-dimensional chirality matrix and charge conjugation matrix are computed as

$$
\hat{\Gamma}_{10}=\left(\begin{array}{cc}
\hat{\gamma} & 0 \\
0 & -\hat{\gamma}
\end{array}\right), \quad C_{10}=\left(\begin{array}{cc}
0 & -C_{4} \hat{\gamma} \\
-C_{4} \hat{\gamma} & 0
\end{array}\right) .
$$

A ten-dimensional Majorana-Weyl fermion (2.1) is decomposed as

$$
\lambda=\left(\begin{array}{c}
\lambda_{+} \mathbf{A} \\
\lambda_{-}^{\mathbf{A}}
\end{array}\right), \quad \hat{\gamma} \lambda_{ \pm}= \pm \lambda_{ \pm}, \quad \lambda_{-}^{\mathbf{A}}=C_{4}\left(\overline{\lambda_{+\mathbf{A}}}\right)^{T}, \quad \overline{\lambda_{-}^{\mathbf{A}}}=-\lambda_{+\mathbf{A}}^{T} C_{4}
$$

The SYM Lagrangian in ten dimensions (2.3) reduces to

$$
\begin{aligned}
\mathcal{L}^{\mathcal{N}=4}=\frac{1}{g_{4}^{2}} \operatorname{Tr}[ & \frac{1}{4} F_{\mu \nu} F^{\mu \nu}-\frac{1}{2} D_{\mu} X_{\mathbf{A B}} D^{\mu} X^{\mathbf{A B}}+\frac{1}{4}\left[X_{\mathbf{A B}}, X_{\mathbf{C D}}\right]\left[X^{\mathbf{A B}}, X^{\mathbf{C D}}\right] \\
& \left.+\overline{\lambda_{+\mathbf{A}}} \not D \lambda_{+\mathbf{A}}+\overline{\lambda_{+\mathbf{C}}}\left[i X_{\mathbf{C D}}, C_{4}\left(\overline{\lambda_{+\mathbf{D}}}\right)^{T}\right]-\lambda_{+\mathbf{A}}^{T} C_{4}\left[i X^{\mathbf{A B}}, \lambda_{+\mathbf{B}}\right]\right] .
\end{aligned}
$$

The supersymmetry transformation rule is

$$
\begin{aligned}
\Delta A_{\mu} & =\overline{\epsilon_{+} \mathbf{A}} \gamma_{\mu} \lambda_{+\mathbf{A}}-\epsilon_{+\mathbf{A}}^{T} \gamma_{\mu}^{T}{\overline{\lambda_{+} \mathbf{A}}}^{T}, \\
\Delta X^{\mathbf{A B}} & =\varepsilon^{\mathbf{A B C D}} \epsilon_{+\mathbf{C}}^{T} C_{4} \lambda_{+\mathbf{D}}+\overline{\epsilon_{+\mathbf{A}}} C_{4}\left(\overline{\lambda_{+\mathbf{B}}}\right)^{T}-\overline{\epsilon_{+\mathbf{B}}} C_{4}\left(\overline{\lambda_{+} \mathbf{A}}\right)^{T},
\end{aligned}
$$




$$
\Delta \lambda_{+\mathbf{A}}=\frac{1}{2} F_{\mu \nu} \gamma^{\mu \nu} \epsilon_{+\mathbf{A}}-2 i \not D X_{\mathbf{A B}} C_{4}\left(\overline{\epsilon_{+} \mathbf{B}}\right)^{T}-2\left[X_{\mathbf{A B}}, X^{\mathbf{B C}}\right] \epsilon_{+\mathbf{C}}
$$

where we used

$$
\epsilon=\left(\begin{array}{c}
\epsilon_{+} \mathbf{A} \\
\epsilon_{-}^{\mathbf{A}}
\end{array}\right), \quad \hat{\gamma} \epsilon_{ \pm}= \pm \epsilon_{ \pm}, \quad \epsilon_{-}^{\mathbf{A}}=-C_{4}\left(\overline{\epsilon_{+} \mathbf{A}}\right)^{T}, \quad \overline{\epsilon_{-}^{\mathbf{A}}}=\epsilon_{+\mathbf{A}}^{T} C_{4} .
$$

Let us perform dimensional reduction for SUSY current. The result is

$$
\begin{aligned}
& \overline{S_{-\mathbf{A}}^{\rho}}=\frac{1}{g_{4}^{2}} \operatorname{Tr}\left[\frac{1}{2} F_{\mu \nu} \overline{\lambda_{+\mathbf{A}}} \gamma^{\rho} \gamma^{\mu \nu}-2 i D_{\mu} X^{\mathbf{B A}} \overline{\lambda_{-}^{\mathbf{B}}} \gamma^{\rho} \gamma^{\mu}+2\left[X_{\mathbf{B C}}, X^{\mathbf{C A}}\right] \overline{\lambda_{+\mathbf{B}}} \gamma^{\rho}\right], \\
& \overline{S_{+}^{\rho \mathbf{A}}}=\frac{1}{g_{4}^{2}} \operatorname{Tr}\left[\frac{1}{2} F_{\mu \nu} \overline{\lambda_{-}^{\mathbf{A}}} \gamma^{\rho} \gamma^{\mu \nu}-2 i D_{\mu} X_{\mathbf{B} \mathbf{A}} \overline{\lambda_{+\mathbf{B}}} \gamma^{\rho} \gamma^{\mu}+2\left[X^{\mathbf{B C}}, X_{\mathbf{C A}}\right] \overline{\lambda_{-}^{\mathbf{B}}} \gamma^{\rho}\right] .
\end{aligned}
$$

$S_{-\mathbf{A}}^{\rho}, S_{+}^{\rho \mathbf{A}}$ are determined so as to satisfy $\overline{\epsilon_{-}^{\mathbf{B}}} S_{+}^{\rho \mathbf{B}}=\overline{S_{-\mathbf{B}}^{\rho}} \epsilon_{+\mathbf{B}}, \overline{\epsilon_{+\mathbf{B}}} S_{-\mathbf{B}}^{\rho}=\overline{S_{+}^{\rho \mathbf{B}}} \epsilon_{-}^{\mathbf{B}}$. Then the supercharge with $\mathrm{SU}(4)$ index is

$$
Q_{-\mathbf{A}}=\int d^{3} x S_{-\mathbf{A}}^{0}, \quad Q_{+}^{\mathbf{A}}=\int d^{3} x S_{+}^{0 \mathbf{A}},
$$

and $\Delta \mathcal{O}=-i\left[\overline{\epsilon_{+\mathbf{B}}} Q_{-\mathbf{B}}+\overline{\epsilon_{-}^{\mathbf{B}}} Q_{+}^{\mathbf{B}}, \mathcal{O}\right]$. The $\mathcal{N}=4$ superalgebra in four dimensions can be computed by dimensional reduction from ten dimension as done in five dimensions. We neglect the contribution of fermions, which is given by total derivative terms and thus vanishes as discussed in the pure $\mathcal{N}=2 \mathrm{SYM}$. The local version of supersymmetry algebra of $\mathcal{N}=4 \mathrm{SYM}$ is

$$
\begin{aligned}
\left\{Q_{-\mathbf{B}}, \overline{S_{\rho-\mathbf{A}}}\right\} & =-2 i T_{\rho \mu} \delta_{\mathbf{B}}^{\mathbf{A}} \gamma^{\mu}+j_{\mathbf{B} \rho \mu}^{\mathbf{A}} \gamma^{\mu}, \\
\left\{Q_{+}^{\mathbf{B}}, \overline{S_{\rho-\mathbf{A}}}\right\} & =-2 i T_{\rho}^{\mathbf{B A}}+j_{\rho}^{\mathbf{B A}}+j_{\rho \sigma \lambda}^{\mathbf{B A}} \gamma^{\lambda \sigma}, \\
\left\{Q_{+}^{\mathbf{B}}, \overline{S_{\rho+}^{\mathbf{A}}}\right\} & =-2 i T_{\rho \mu} \delta_{\mathbf{A}}^{\mathbf{B}} \gamma^{\mu}-j_{\mathbf{A} \rho \mu}^{\mathbf{B}} \gamma^{\mu}, \\
\left\{Q_{-\mathbf{B}}, \overline{S_{\rho+}^{\mathbf{A}}}\right\} & =-2 i T_{\rho \mathbf{B A}}-j_{\rho \mathbf{B A}}-j_{\mathbf{B A} \rho \sigma \lambda} \gamma^{\lambda \sigma},
\end{aligned}
$$

where

$$
\begin{aligned}
T_{\mu \rho}= & \frac{1}{4 g_{4}^{2}} \operatorname{Tr}\left[g_{\mu \rho}\left(F_{\nu \sigma} F^{\nu \sigma}-2 D_{\nu} X_{\mathbf{A B}} D^{\nu} X^{\mathbf{A B}}+\left[X_{\mathbf{A B}}, X_{\mathbf{C D}}\right]\left[X^{\mathbf{A B}}, X^{\mathbf{C D}}\right]\right)\right. \\
& \left.+4\left(F_{\mu}{ }^{\nu} F_{\nu \rho}+D_{\mu} X^{\mathbf{A B}} D_{\rho} X_{\mathbf{A B}}\right)\right] \\
T_{\rho \mathbf{A B}}= & \frac{2 i}{g_{4}^{2}} \partial_{\nu} \operatorname{Tr}\left[F_{\rho}^{\nu} X_{\mathbf{A B}}\right] \\
j_{\mathbf{B} \rho \mu}^{\mathbf{A}}= & -\frac{4 i}{g_{4}^{2}} \sigma_{\rho \sigma \nu \mu} \partial^{\sigma} \operatorname{Tr}\left[X_{\mathbf{B C}} D^{\nu} X^{\mathbf{C A}}\right], \\
j_{\rho}^{\mathbf{B A}}= & -\frac{2}{g_{4}^{2}} \sigma_{\rho}^{\sigma \mu \nu} \partial_{\sigma} \operatorname{Tr}\left[X^{\mathbf{B A}} F_{\mu \nu}\right], \\
j_{\rho \sigma \lambda}^{\mathbf{B A}}= & -\frac{4}{3 g_{4}^{2}} \sigma_{\rho \nu \sigma \lambda} \partial^{\nu} \operatorname{Tr}\left[\left[X^{\mathbf{B C}}, X_{\mathbf{C D}}\right], X^{\mathbf{D A}}\right] .
\end{aligned}
$$


Volume integration of both sides leads to supersymmetry algebra in $\mathcal{N}=4$ SYM.

$$
\begin{aligned}
\left\{Q_{-\mathbf{B}}, \overline{Q_{-\mathbf{A}}}\right\} & =-2 i P_{\mu} \delta_{\mathbf{B}}^{\mathbf{A}} \gamma^{\mu}+Z_{\mathbf{B} \mu}^{\mathbf{A}} \gamma^{\mu}, \\
\left\{Q_{+}^{\mathbf{B}}, \overline{Q_{-\mathbf{A}}}\right\} & =-2 i P^{\mathbf{B A}}+Z^{\mathbf{B A}}+Z_{\sigma \lambda}^{\mathbf{B A}} \gamma^{\lambda \sigma}, \\
\left\{Q_{+}^{\mathbf{B}}, \overline{Q_{+}^{\mathbf{A}}}\right\} & =-2 i P_{\mu} \delta_{\mathbf{A}}^{\mathbf{B}} \gamma^{\mu}-Z_{\mathbf{A} \mu}^{\mathbf{B}} \gamma^{\mu}, \\
\left\{Q_{-\mathbf{B}}, \overline{Q_{+}^{\mathbf{A}}}\right\} & =-2 i P_{\mathbf{B A}}-Z_{\mathbf{B A}}-Z_{\mathbf{B A} \sigma \lambda} \gamma^{\lambda \sigma},
\end{aligned}
$$

where we set

$$
\begin{aligned}
& P^{\mu}=\int d^{3} x T^{0 \mu}, \quad P^{\mathbf{A B}}=\int d^{3} x T^{0 \mathbf{A B}}, \\
& Z_{\mathbf{B} \mu}^{\mathbf{A}}=\int d^{3} x j_{\mathbf{B}}^{\mathbf{A} 0}{ }_{\mu}, \quad Z^{\mathbf{B A}}=\int d^{3} x j^{\mathbf{B A} 0}, \quad Z_{\lambda \sigma}^{\mathbf{A B}}=\int d^{3} x j^{\mathbf{A B} 0}{ }_{\lambda \sigma} .
\end{aligned}
$$

As an example, let us consider the case of SU(2) gauge group in the Coulomb branch.

$$
X_{12}=\frac{a}{\sqrt{2}} \sigma_{3}, \quad X_{13}=X_{14}=0
$$

Then (5.83) is computed as

$$
\left\{Q_{-1}, \overline{Q_{+}^{2}}\right\}=\left\{Q_{-3}, \overline{Q_{+}^{4}}\right\}=2 \sqrt{2} \mathcal{Z}
$$

where

$$
\mathcal{Z}=n_{e} a+n_{m} a_{D}
$$

with $n_{e}, n_{m}$ given by (5.30), $a_{D}=\tau_{0} a, \tau_{0}=\frac{4 \pi i}{g_{\perp}^{2}}$. The formula of the central charge with normalization of the holomorphic coupling in $\mathcal{N}^{4}=4 \mathrm{SYM}$ is the same as pure $\mathcal{N}=2 \mathrm{SYM}$, which is again consistent with the result in [29].

\section{Discussion}

We have determined supersymmetry algebra of SYM of a vector multiplet in six dimensions including the contribution of fermions, which is given by boundary terms. We have extended this calculation to the case including a hyper multiplet. For SUSY algebra of six dimensional maximally SYM we have carried out dimensional reduction for that in ten dimensions. From six dimensional results we have performed dimensional reduction to determine SUSY algebras of five and four dimensional SYM. From six to five the KaluzaKlein momentum arising from torus compactification is different from the instanton-particle charge though they are indistinguishable in the superalgebra. And the Kaluza-Klein momentum corresponds to the electric charge part in the famous formula of central charge. We have derived the whole extended supersymmetry algebra as well as the holomorphic coupling constant introduced in [29] against the four dimensional $\mathcal{N}=2 \mathrm{SYM}$ including fundamental hyper multiplets and $\mathcal{N}=4 \mathrm{SYM}$.

Since we started from SYM in six dimensions with the canonical kinetic term in this paper, the theory obtained by dimensional reduction inherited this property. Computing SUSY algebra of general SYM with the non-canonical kinetic term is left to future work, 
though the general structure of the algebra will remain unchanged. Especially in five and four dimensions a general Lagrangian contains topological terms such as Chern-Simons term and $F \wedge F$, respectively, which has an extra effect on physics of the theory [43].

It should be possible to determine BPS states in maximally SYM in six dimensions in Higgs branch. In terms of brane picture, maximally SYM in six dimensions is realized on D-five branes, and Higgs branch corresponds to separation thereof. Then BPS states on this branch will correspond to supersymmetric brane configuration realized on the separated D-five branes set up. It would be interesting to clarify relations between those BPS states in six dimensional SYM and those in five dimensional maximally SYM in broken phase, which has close relationship with the $(2,0)$ theory describing M-five branes [18].

We hope to come back to these problems in the future.

\section{Acknowledgments}

The author was supported by the Israeli Science Foundation under grant 352/13 and $504 / 13$.

\section{A A formula of gamma matrix}

In this appendix, we derive a formula of gamma matrix given in appendix of [44]. We use notation such that

$$
\Gamma^{\mu_{0} \cdots \mu_{n}}=\Gamma^{\left[\mu_{0}\right.} \cdots \Gamma^{\left.\mu_{n}\right]}=\frac{1}{(n+1) !} \sum_{\sigma \in \mathfrak{S}_{n+1}}(-)^{\sigma} \Gamma^{\mu_{\sigma(0)}} \cdots \Gamma^{\mu_{\sigma(n)}}
$$

where $\mathfrak{S}_{N}$ is the set of permutation of $N$ elements.

Denoting $C_{n, m}, D_{n, m}$ by

$$
\begin{aligned}
& \Gamma^{M_{1} \cdots M_{m}} \Gamma^{N_{1} \cdots N_{n}} \Gamma_{M_{m} \cdots M_{1}}=C_{n, m} \Gamma^{N_{1} \cdots N_{n}}, \\
&(-)^{m-1} \Gamma^{M_{1} \cdots M_{m-1}\left[N_{1}\right.} \Gamma^{\left.N_{2} \cdots N_{n}\right]} \Gamma_{M_{m-1} \cdots M_{1}}=D_{n, m} \Gamma^{N_{1} \cdots N_{n}},
\end{aligned}
$$

we can relate $C_{n, m}, D_{n, m}$ by

$$
\begin{aligned}
C_{n, m} & =(-)^{m} C_{n-1, m}+2 m(-)^{m+n} D_{n-1, m-1}, \\
D_{n, m} & =\frac{1}{2}\left(C_{n, m}+(-)^{m} C_{n+1, m}\right) .
\end{aligned}
$$

From these we find

$$
C_{n, m}=(-)^{m} C_{n-1, m}+m(-)^{m+n} C_{n-1, m-1}+m(-)^{n+1} C_{n, m-1} .
$$

One can easily check that

$$
C_{n, 0}=1, \quad C_{0, m}=D(D-1) \cdots(D-(m-1)),
$$

where $D$ is an arbitrary dimension. By using (A.6) and (A.7) one can determine $C_{n, m}$ (and thus $\left.D_{n, m}\right)$ inductively. As examples, we determine $C_{n, m}$ when $D=6,10$. The result of $D=6$ matches that given in [44]. 


\begin{tabular}{|c|c|c|c|c|}
\hline$m=3$ & 120 & 0 & -24 & 0 \\
\hline$m=2$ & 30 & 10 & -2 & -6 \\
\hline$m=1$ & 6 & -4 & 2 & 0 \\
\hline$m=0$ & 1 & 1 & 1 & 1 \\
\hline$C_{n, m}$ & $n=0$ & $n=1$ & $n=2$ & $n=3$ \\
\hline
\end{tabular}

Table 1. $D=6$.

\begin{tabular}{|c|c|c|c|c|c|c|}
\hline$m=5$ & 30240 & 0 & -3360 & 0 & 1440 & 0 \\
\hline$m=4$ & 5040 & 1008 & -336 & -336 & 48 & 240 \\
\hline$m=3$ & 720 & -288 & 48 & 48 & -48 & 0 \\
\hline$m=2$ & 90 & 54 & 26 & 6 & -6 & -10 \\
\hline$m=1$ & 10 & -8 & 6 & -4 & 2 & 0 \\
\hline$m=0$ & 1 & 1 & 1 & 1 & 1 & 1 \\
\hline$C_{n, m}$ & $n=0$ & $n=1$ & $n=2$ & $n=3$ & $n=4$ & $n=5$ \\
\hline
\end{tabular}

Table 2. $D=10$.

\section{B Convention in six dimensions}

In this appendix we collect convention in six dimensions used in this paper. We realize $\mathrm{SO}(1,5)$ matrices satisfying $\left\{\Gamma_{M}, \Gamma_{N}\right\}=2 g_{M N}$, where $\left(g_{M N}\right)=\operatorname{diag}(-1,1, \cdots, 1)$, in two ways. One is

$$
\Gamma_{\mu}=\gamma_{\mu} \otimes \sigma_{1}, \quad \Gamma_{5}=\mathbf{1} \otimes \sigma_{2}
$$

where $\gamma^{\mu}(\mu=0,1,2,3)$ are $\mathrm{SO}(1,3)$ matrices realized as

$$
\gamma_{\mu}=\left(\begin{array}{cc}
0 & \bar{\sigma}_{\mu}^{\prime} \\
\sigma_{\mu}^{\prime} & 0
\end{array}\right),
$$

where $\bar{\sigma}_{0}^{\prime}=\sigma_{0}^{\prime}=i \sigma_{2}, \bar{\sigma}_{1}^{\prime}=\sigma_{1}^{\prime}=\sigma_{1}, \bar{\sigma}_{2}^{\prime}=\sigma_{2}^{\prime}=\sigma_{3}, \bar{\sigma}_{3}^{\prime}=-\sigma_{3}^{\prime}=i$ with $\sigma_{i}$ Pauli matrices satisfying $\sigma_{i} \sigma_{j}=\delta_{i j}+i \varepsilon_{i j k} \sigma_{k}$. This realization is useful when we consider dimensional reduction from six dimensions to five ones. The other is

$$
\Gamma_{\mu}=\gamma_{\mu} \otimes \mathbf{1}, \quad \Gamma_{4}=\hat{\gamma} \otimes \sigma_{1}, \quad \Gamma_{5}=\hat{\gamma} \otimes \sigma_{2}
$$

where $\hat{\gamma}=i \gamma_{0123}$ is a chirality matrix in four dimensions. This is convenient when we do dimensional reduction from six to four. In both cases, we define the charge conjugation matrix as $C_{6}=\Gamma_{035}$, which satisfies

$$
C_{6}^{2}=1, \quad C_{6}^{*}=C_{6}, \quad C_{6}^{T}=C_{6}, \quad C_{6} \Gamma^{M}=-\left(\Gamma^{M}\right)^{T} C_{6} .
$$

In Lorentzian six dimensions there exists a symplectic majorana Weyl spinor. By denoting $\operatorname{Sp}(1)$-Majorana fermion by $\lambda^{A}$ it satisfies

$$
\lambda^{A}=\varepsilon^{A B} C_{6}{\overline{\lambda^{B}}}^{T} .
$$


Two symplectic Majorana fermions $\psi^{A}, \chi^{A}$ satisfy

$$
\overline{\psi^{A}} \gamma_{\mu_{1}} \cdots \gamma_{\gamma_{k}} \chi^{B}=(-)^{k+1}\left(\overline{\chi^{A}} \gamma_{\mu_{k}} \cdots \gamma_{\gamma_{1}} \psi^{B}-\delta_{A}^{B} \overline{\chi^{D}} \gamma_{\mu_{k}} \cdots \gamma_{\gamma_{1}} \psi^{D}\right) .
$$

Especially taking trace in terms of $\mathrm{Sp}(1)$ index gives

$$
\overline{\psi^{A}} \gamma_{\mu_{1}} \cdots \gamma_{\gamma_{k}} \chi^{A}=(-)^{k} \overline{\chi^{A}} \gamma_{\mu_{k}} \cdots \gamma_{\gamma_{1}} \psi^{A}
$$

Open Access. This article is distributed under the terms of the Creative Commons Attribution License (CC-BY 4.0), which permits any use, distribution and reproduction in any medium, provided the original author(s) and source are credited.

\section{References}

[1] L. Brink, J.H. Schwarz and J. Scherk, Supersymmetric Yang-Mills theories, Nucl. Phys. B 121 (1977) 77 [inSPIRE].

[2] E. Witten, Phase transitions in M-theory and F-theory, Nucl. Phys. B 471 (1996) 195 [hep-th/9603150] [INSPIRE].

[3] N. Seiberg, Five-dimensional SUSY field theories, nontrivial fixed points and string dynamics, Phys. Lett. B 388 (1996) 753 [hep-th/9608111] [INSPIRE].

[4] O. Aharony and A. Hanany, Branes, superpotentials and superconformal fixed points, Nucl. Phys. B 504 (1997) 239 [hep-th/9704170] [INSPIRE].

[5] O. Aharony, A. Hanany and B. Kol, Webs of $(p, q)$ five-branes, five-dimensional field theories and grid diagrams, JHEP 01 (1998) 002 [hep-th/9710116] [INSPIRE].

[6] O. DeWolfe, A. Hanany, A. Iqbal and E. Katz, Five-branes, seven-branes and five-dimensional $E_{n}$ field theories, JHEP 03 (1999) 006 [hep-th/9902179] [INSPIRE].

[7] F. Benini, S. Benvenuti and Y. Tachikawa, Webs of five-branes and $N=2$ superconformal field theories, JHEP 09 (2009) 052 [arXiv:0906.0359] [INSPIRE].

[8] O. Bergman, D. Rodríguez-Gómez and G. Zafrir, 5-brane webs, symmetry enhancement and duality in 5d supersymmetric gauge theory, JHEP 03 (2014) 112 [arXiv:1311.4199] [INSPIRE].

[9] H.-C. Kim, S.-S. Kim and K. Lee, 5-dim superconformal index with enhanced $E_{n}$ global symmetry, JHEP 10 (2012) 142 [arXiv:1206.6781] [INSPIRE].

[10] D. Bashkirov, A comment on the enhancement of global symmetries in superconformal $\mathrm{SU}(2)$ gauge theories in $5 D$, arXiv: 1211.4886 [INSPIRE].

[11] M. Taki, Notes on enhancement of flavor symmetry and $5 d$ superconformal index, arXiv: 1310.7509 [INSPIRE].

[12] O. Bergman, D. Rodríguez-Gómez and G. Zafrir, Discrete $\theta$ and the $5 d$ superconformal index, JHEP 01 (2014) 079 [arXiv:1310.2150] [INSPIRE].

[13] O. Bergman, D. Rodríguez-Gómez and G. Zafrir, $5 d$ superconformal indices at large- $N$ and holography, JHEP 08 (2013) 081 [arXiv: 1305.6870] [INSPIRE].

[14] Y. Tachikawa, Instanton operators and symmetry enhancement in $5 d$ supersymmetric gauge theories, Prog. Theor. Exp. Phys. 2015 (2015) 043B06 [arXiv:1501.01031] [INSPIRE]. 
[15] G. Zafrir, Instanton operators and symmetry enhancement in 5d supersymmetric USp, SO and exceptional gauge theories, JHEP 07 (2015) 087 [arXiv:1503.08136] [INSPIRE].

[16] K. Yonekura, Instanton operators and symmetry enhancement in $5 d$ supersymmetric quiver gauge theories, JHEP 07 (2015) 167 [arXiv:1505.04743] [INSPIRE].

[17] M.R. Douglas, On D = 5 super Yang-Mills theory and (2,0) theory, JHEP 02 (2011) 011 [arXiv: 1012.2880] [INSPIRE].

[18] N. Lambert, C. Papageorgakis and M. Schmidt-Sommerfeld, M5-branes, D4-branes and quantum 5D super-Yang-Mills, JHEP 01 (2011) 083 [arXiv:1012.2882] [INSPIRE].

[19] Z. Bern, J.J. Carrasco, L.J. Dixon, M.R. Douglas, M. von Hippel and H. Johansson, $D=5$ maximally supersymmetric Yang-Mills theory diverges at six loops,

Phys. Rev. D 87 (2013) 025018 [arXiv: 1210.7709] [InSPIRE].

[20] H.-C. Kim, S. Kim, E. Koh, K. Lee and S. Lee, On instantons as Kaluza-Klein modes of M5-branes, JHEP 12 (2011) 031 [arXiv:1110.2175] [INSPIRE].

[21] H.-C. Kim, J. Kim and S. Kim, Instantons on the 5-sphere and M5-branes, arXiv: 1211.0144 [INSPIRE].

[22] N. Seiberg, Nontrivial fixed points of the renormalization group in six-dimensions, Phys. Lett. B 390 (1997) 169 [hep-th/9609161] [INSPIRE].

[23] U.H. Danielsson, G. Ferretti, J. Kalkkinen and P. Stjernberg, Notes on supersymmetric gauge theories in five-dimensions and six-dimensions, Phys. Lett. B 405 (1997) 265 [hep-th/9703098] [INSPIRE].

[24] O.J. Ganor and A. Hanany, Small E $E_{8}$ instantons and tensionless noncritical strings, Nucl. Phys. B 474 (1996) 122 [hep-th/9602120] [inSPIRE].

[25] N. Seiberg and E. Witten, Comments on string dynamics in six-dimensions, Nucl. Phys. B 471 (1996) 121 [hep-th/9603003] [INSPIRE].

[26] M.J. Duff, H. Lü and C.N. Pope, Heterotic phase transitions and singularities of the gauge dyonic string, Phys. Lett. B 378 (1996) 101 [hep-th/9603037] [INSPIRE].

[27] O.J. Ganor, Six-dimensional tensionless strings in the large- $N$ limit, Nucl. Phys. B 489 (1997) 95 [hep-th/9605201] [INSPIRE].

[28] E. Witten, New 'gauge' theories in six-dimensions, JHEP 01 (1998) 001 [hep-th/9710065] [INSPIRE].

[29] N. Seiberg and E. Witten, Monopoles, duality and chiral symmetry breaking in $N=2$ supersymmetric QCD, Nucl. Phys. B 431 (1994) 484 [hep-th/9408099] [INSPIRE].

[30] H. Osborn, Topological charges for $N=4$ supersymmetric gauge theories and monopoles of spin 1, Phys. Lett. B 83 (1979) 321 [InSPIRE].

[31] I.A. Popescu and A.D. Shapere, BPS equations, BPS states and central charge of $N=2$ supersymmetric gauge theories, JHEP 10 (2002) 033 [hep-th/0102169] [INSPIRE].

[32] T.T. Dumitrescu and N. Seiberg, Supercurrents and brane currents in diverse dimensions, JHEP 07 (2011) 095 [arXiv:1106.0031] [INSPIRE].

[33] N. Seiberg and E. Witten, Electric-magnetic duality, monopole condensation and confinement in $N=2$ supersymmetric Yang-Mills theory, Nucl. Phys. B 426 (1994) 19 [Erratum ibid. B 430 (1994) 485] [hep-th/9407087] [INSPIRE]. 
[34] E. Witten, Solutions of four-dimensional field theories via M-theory, Nucl. Phys. B 500 (1997) 3 [hep-th/9703166] [INSPIRE].

[35] D. Gaiotto, $N=2$ dualities, JHEP 08 (2012) 034 [arXiv:0904.2715] [INSPIRE].

[36] R. Jackiw and C. Rebbi, Solitons with fermion number 1/2, Phys. Rev. D 13 (1976) 3398 [INSPIRE].

[37] C.R. Nohl, Bound state solutions of the Dirac equation in extended hadron models, Phys. Rev. D 12 (1975) 1840 [INSPIRE].

[38] C. Callias, Index theorems on open spaces, Commun. Math. Phys. 62 (1978) 213 [InSPIRE].

[39] M.F. Atiyah, N.J. Hitchin, V.G. Drinfeld and Yu. I. Manin, Construction of instantons, Phys. Lett. A 65 (1978) 185 [inSPIRE].

[40] N. Dorey, T.J. Hollowood, V.V. Khoze and M.P. Mattis, The calculus of many instantons, Phys. Rept. 371 (2002) 231 [hep-th/0206063] [INSPIRE].

[41] E. Witten and D.I. Olive, Supersymmetry algebras that include topological charges, Phys. Lett. B 78 (1978) 97 [INSPIRE].

[42] P.C. Argyres and N. Seiberg, S-duality in $N=2$ supersymmetric gauge theories, JHEP 12 (2007) 088 [arXiv:0711.0054] [INSPIRE].

[43] E. Witten, Dyons of charge e $\theta / 2 \pi$, Phys. Lett. B 86 (1979) 283 [INSPIRE].

[44] T. Kugo and K. Ohashi, Supergravity tensor calculus in $5 D$ from $6 D$, Prog. Theor. Phys. 104 (2000) 835 [hep-ph/0006231] [INSPIRE]. 